\title{
NON-DYADIC WAVELET ANALYSIS
}

\author{
D.S.G. POLLOCK and IOLANDA LO CASCIO \\ Department of Economics, Queen Mary College, \\ University of London, \\ Mile End Road, London E1 4NS, UK
}

\begin{abstract}
The conventional dyadic multiresolution analysis constructs a succession of frequency intervals in the form of $\left(\pi / 2^{j}, \pi / 2^{j-1}\right) ; j=1,2, \ldots, n$ of which the bandwidths are halved repeatedly in the descent from high frequencies to low frequencies. Whereas this scheme provides an excellent framework for encoding and transmitting signals with a high degree of data compression, it is less appropriate to statistical data analysis.

This paper describes a non-dyadic mixed-radix wavelet analysis which allows the wave bands to be defined more flexibly than in the case of a conventional dyadic analysis. The wavelets that form the basis vectors for the wave bands are derived from the Fourier transforms of a variety of functions that specify the frequency responses of the filters corresponding to the sequences of wavelet coefficients.
\end{abstract}

\section{Introduction: Dyadic and Non-Dyadic Wavelet Analysis}

The statistical analysis of time series can be pursued either in the time domain or in the frequency domain, or in both. A time-domain analysis will reveal the sequence of events within the data, so long as the events do not coincide. A frequency-domain analysis, which describes the data in terms of sinusoidal functions, will reveal its component sequences, whenever they subsist in separate frequency bands. The analyses in both domains are commonly based on the assumption of stationarity. If the assumption is not satisfied, then, often, a transformation can be applied to the data to make them resemble a stationary series. For a stationary series, the results that are revealed in one domain can be transformed readily into equivalent results in the other domain.

The revolution in statistical Fourier analysis that occurred in the middle of the twentieth century established the equivalence of the two domains under the weak assumption of statistical stationarity. Previously, it had seemed that frequency-domain analysis was fully applicable only to strictly periodic functions of a piecewise continuous nature. However, the additional flexibility of statistical Fourier analysis is not sufficient to cope with 


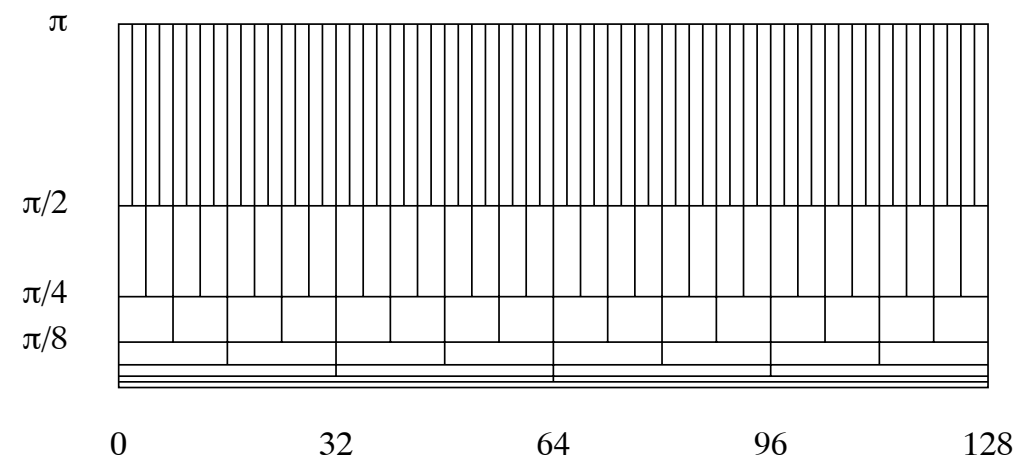

Figure 1. The partitioning of the time-frequency plane according to a dyadic multiresolution analysis of a data sequence of $T=128=2^{7}$ points.

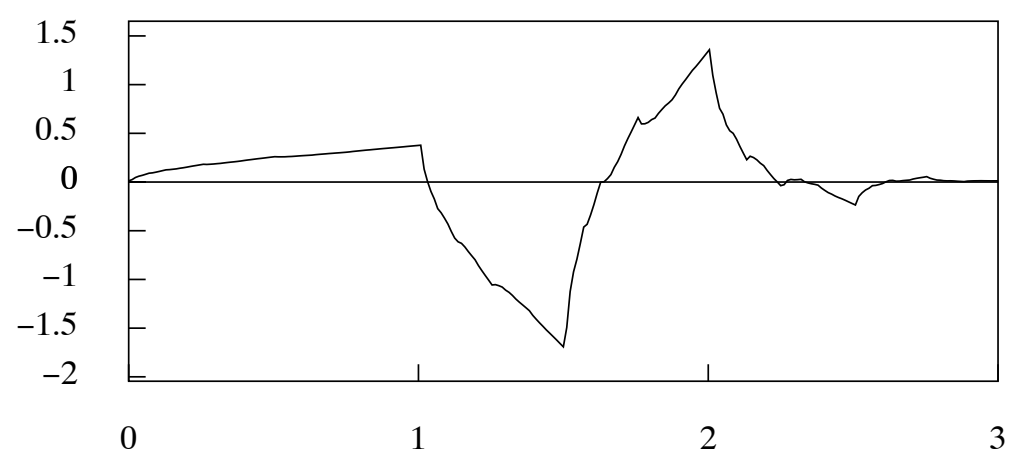

Figure 2. The Daubechies D4 wavelet function calculated via a recursive method.

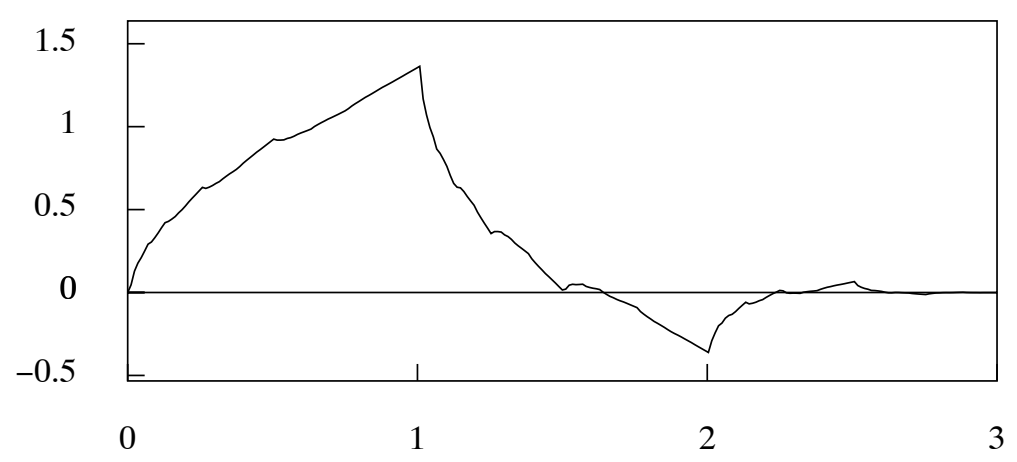

Figure 3. The Daubechies D4 scaling function calculated via a recursive method. 
phenomena that are truly evolving through time. A sufficient flexibility to deal with evolutionary phenomena can be achieved by combining the time domain and the frequency domain in a so-called wavelet analysis.

The replacement of classical Fourier analysis by wave packet analysis occurred in the realms of quantum mechanics many years ago when Schrödinger's time-dependent wave equation became the model for all sorts of electromagnetic phenomena. (See Dirac 1958, for example.) This was when the dual wave-particle analogy of light superseded the classical wave analogy that had displaced the ancient corpuscular theory. It is only recently, at the end of the twentieth century, that formalisms that are similar to those of quantum mechanics have penetrated statistical time-series analysis. The result has been the new and rapidly growing field of wavelet analysis.

The common form of dyadic wavelet analysis entails a partition of the time-frequency plane of the sort that is depicted in Figure 1, which relates to the wavelet analysis of a sample of $T=2^{7}=128$ points. The wavelets are functions of continuous time that reside in a succession of horizontal frequency bands. Each band contains a succession of wavelets, distributed over time, of which the centres lie in the cells that partition the band. Within a given band, the wavelets have a common frequency content and a common temporal dispersion, but their amplitude, which is their vertical scale, is free to vary. As we proceed down the frequency scale from one band to the next, the bandwidth of the frequencies is halved and the temporal dispersion of the wavelets, which is reflected in the width of the cells, is doubled.

The wavelet bands are created by a recursive process of subdivision. In the first round, the frequency range is divided in two. The upper band $[\pi / 2, \pi]$ is populated by $T / 2$ wavelets, separated, one from the next, by two sampling intervals, and the lower band $[0, \pi / 2]$ is populated by the same number of scaling functions in a similar sequence. Thus, there are as many functions as there are data points. In the next round, the lower half of the frequency range is subdivided into an upper band $[\pi / 4, \pi / 2]$ of wavelets and a lower band $[0, \pi / 4]$ of scaling functions, with both bands containing $T / 4$ functions, separated by four intervals. The process can be repeated such that, in the $j$ th round, the $j$ th band is divided into an upper band of wavelets and a lower hand of scaling functions, with $T / 2^{j}$ functions in each. If that number is no longer divisible by 2 , then the process must terminate. However, if $T=2^{n}$, as is the case for Figure 1, then it can be continued through $n$ rounds until the $n$th band contains a single wavelet, and there is a constant function to accompany it in place of a scaling function.

The object of the wavelet analysis is to associate an amplitude coefficient to each of the wavelets. The variation in the amplitude coefficients enables 
a wavelet analysis to reflect the changing structure of a non-stationary time series. By contrast, the amplitude coefficients that are associated with the sinusoidal basis functions of a Fourier analysis remain constant throughout the sample.(Accounts of wavelet analysis, which place it within the context of Fourier analysis, have been given by Newland (1993) and by Boggess and Narcowich (2001). Other accessible accounts have been given by Burrus, Gopinath and Guo (1998) and by Misiti, Misiti, Oppenheim and Poggi (1997) in the user's guide to the MATLAB Wavelets Toolbox.)

The wavelets that are employed within the dyadic scheme are usually designed to be mutually orthogonal. They can be selected from a wide range of wavelet families. The most commonly employed wavelets are from the Daubechies (1988), (1992) family. Figures 2 and 3 display the level-1 D4 Daubechies wavelet and scaling function, which are generated on the first division of the time-frequency plane, and which span the upper and the lower halves of the frequency range $[0, \pi]$, respectively. These are highly localised continuous functions of a fractal nature that have finite supports with a width of three sampling intervals. The Daubechies wavelets have no available analytic forms, and they are not readily available in sampled versions. They are defined, in effect, by the associated dilation coefficients. These express a wavelet in one frequency band and a scaling function in the band below - which has the same width and which stretches to zero - as a linear combination of the more densely packed and less dispersed scaling functions that form a basis for the two bands in combination.

The fact that the Daubechies wavelets are know only via their dilation coefficients is no impediment to the discrete wavelet transform. This transform generates the amplitude coefficients associated with the wavelet decomposition of a data sequence; and it is accomplished via the pyramid algorithm of Mallat (1989). The continuous-time wavelets are, in reality, a shadowy accompaniment - and, in some ways, an inessential one - of a discrete-time analysis that can be recognised as an application of the techniques of multi-rate filtering, which are nowadays prevalent in communications engineering. (For examples, see Vaidyanathan 1993, Strang and Nguyen 1997 and Vetterli and Kovacević 1995.) In this perspective, the dilation coefficients of the wavelets and of the associated scaling functions are nothing but the coefficients of a pair of quadrature mirror filters that are applied in successive iterations of the pyramid algorithm. This uncommon relationship between the continuous-time and the discrete-time aspects of the analysis is undoubtedly the cause of many conceptual difficulties.

The Daubechies-Mallat paradigm has been very successful in application to a wide range of signal processing problems, particularly in audioacoustic analysis and in the analysis of digitised picture images, which are two-dimensional signals in other words. There are at least two reasons for 


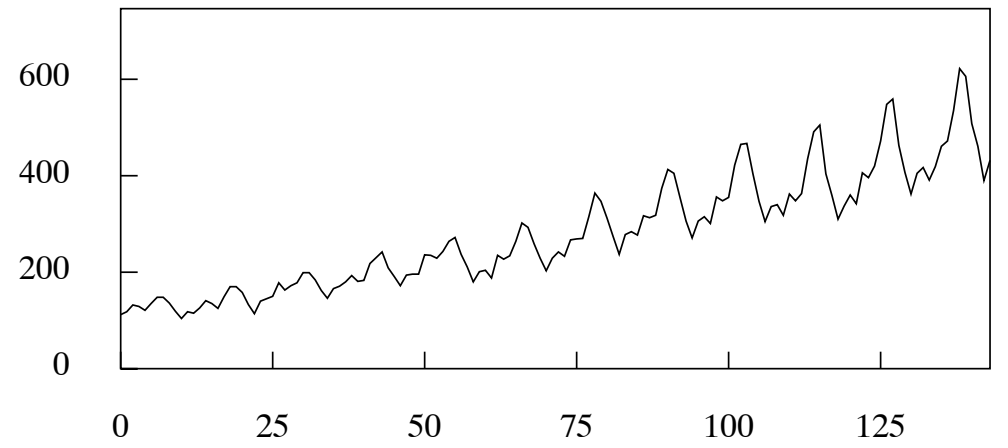

Figure 4. International airline passengers: monthly totals (thousands of passengers) January 1949-December 1960: 144 observations.

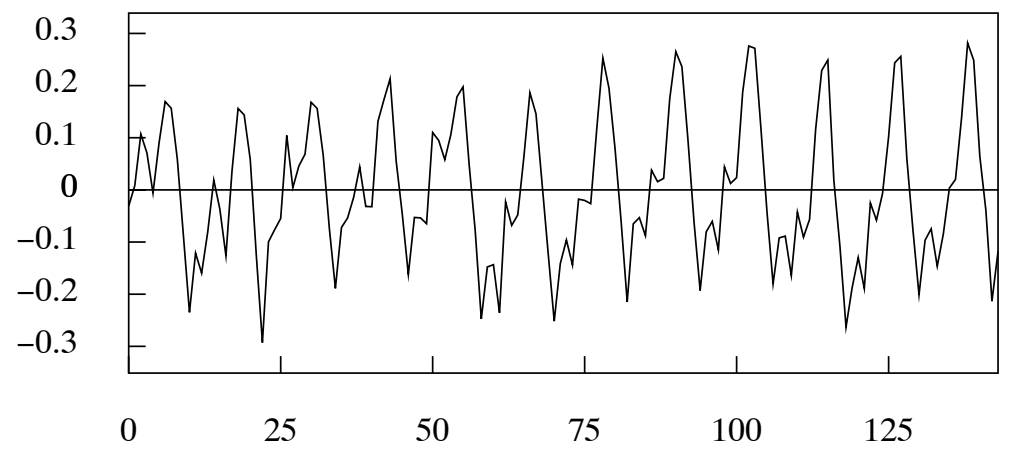

Figure 5. The seasonal fluctuation in the airline passenger series, represented by the residuals from fitting a quadratic function to the logarithms of the series.

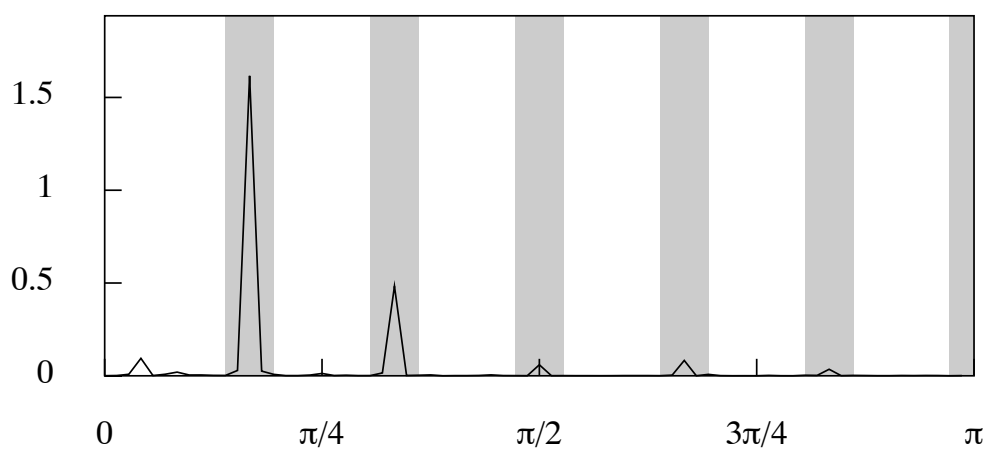

Figure 6. The periodogram of the seasonal fluctuations in the airline passenger series. 
this success. The first concerns the efficiency of the pyramid algorithm, which is ideal for rapid processing in real time. The second reason lies in the Daubechies wavelets themselves. Their restricted supports are a feature that greatly assists the computations. This feature, allied to the sharp peaks of the wavelets, also assists in the detection of edges and boundaries in images.

The system of Daubechies and Mallat is not suited to all aspects of statistical signal extraction. For a start, the Daubechies wavelets might not be the appropriate ones to select. Their disjunct nature can contrast with the smoother and more persistent motions that underlie the data. The non-availability of their discretely sampled versions may prove to be an impediment; and the asymmetric nature of the associated dilation coefficients might conflict with the requirement, which is commonly imposed upon digital filters, that there should be no phase effects. (The absence of phase effects is important when, for example, wavelets are used as an adjunct to transfer-function modelling, as in the investigations of Ramsey and Lampart 1998 and of Nason and Sapatinas 2002.) A more fundamental difficulty lies in the nature of the dyadic decomposition. In statistical analyses, the structures to be investigated are unlikely to fall neatly into dyadic time and frequency bands, such as those of Figure 1; and the frequency bands need to be placed wherever the phenomena of interest happen to be located.

For an example of a statistical data series that requires a more flexible form of wavelet analysis, we might consider the familiar monthly airline passenger data of Box and Jenkins (1976), depicted in Figure 4, which comprises $T=144=3^{2} \times 2^{4}$ data points. The detrended series, which is obtained by taking the residuals from fitting a quadratic function to the logarithms of the data, is shown in Figure 5. The detrended data manifest a clear pattern of seasonality, which is slowly evolving in a manner that is readily intelligible if one thinks of the development of air travel over the period in question - the summer peak in air travel was increasing relative to the winter peak throughout the period. The components of the seasonal pattern lie in and around a set of harmonically related frequencies $\{\pi j / 6 ; j=1, \ldots, 6\}$. This can be seen in Figure 6, which displays the periodogram of the seasonal fluctuations.

In order to capture the evolving seasonal pattern, one might apply a wavelet analysis to some narrow bands surrounding the seasonal frequencies. To isolate bands extending for 5 degrees on either side of the seasonal frequencies, (excepting the frequency of $\pi$, where there is nothing above,) one must begin by dividing the frequency range in $36=3^{2} \times 2^{2}$ equal bands. The requisite wavelets will be obtained by dilating the first-level wavelet by a factor of 3 as well as by the dyadic factor of 2 . These bands are indicated 
on Figure 6. The other choices for the bandwidths would be 6 degrees, $7 \frac{1}{2}$ degrees 10 , degrees and 15 degrees - the latter affording no interstices between the bands.

\section{The Aims of the Paper}

The intention of this paper is to provide the framework for a flexible method of wavelet analysis that is appropriate to nonstationary data that have been generated by evolving structures that fall within non-dyadic frequency bands. For this purpose, we have to consider collections of wavelets and filters that are related to each other by dilation factors in addition to the factor of 2 . At the same time, we shall endeavour to accommodate samples of all sizes, thereby relieving the restriction that $T=2^{n}$, which is necessary for a complete dyadic decomposition.

We shall use the so-called Shannon wavelet as a prototype, since it is readily amenable to dilations by arbitrary factors. Since the Shannon wavelets are defined by a simple analytic function, their sampled versions are readily available; and their ordinates constitute the coefficients of symmetric digital filters that have no phase effects. Thus, in the case of the Shannon wavelets, the connection between the continuous-time analysis and the discrete-time analysis is uniquely straightforward: the sampled ordinates of the wavelets and scaling functions constitute the filter coefficients of the discrete-time analysis, which are also the coefficients of the dilation relationships. The orthogonality conditions that affect the Shannon wavelets are easy to demonstrate. The conditions are important in a statistical analysis, since they enable the testing of hypotheses to proceed on the basis of simple chi-square statistics.

The disadvantage of the Shannon wavelets is in their wide dispersion. They have an infinite support, which is the entire real line. However, they can be adapted to the analysis of a finite data sequence of $T$ points by wrapping their sampled coefficients around a circle of circumference $T$ and by adding the coincident coefficients. The wrapping is achieved by sampling the corresponding energy functions in the frequency domain at regular intervals. The wavelet coefficients in the time domain may be obtained by applying the discrete Fourier transform to the square roots of the ordinates sampled from the energy functions.

The band limitation of the energy functions enhances the efficiency of computations performed in the frequency domain, which entail simple multiplications or modulations. At the same time, it prejudices the efficiency of computations performed in the time domain, which entail the circular convolutions of sequences of length $T$. For this reason, we choose to conduct our filtering operations in the frequency domain. The mixed-radix fast 
Fourier transform of Pollock (1999) may be used to carry the data into the frequency domain; and it may be used, in its inverse mode, to carry the products of the filtering operations back to the time domain.

Despite the availability of these techniques for dealing with finite samples, the wide dispersion of the Shannon wavelets remains one of their significant disadvantages. Therefore, we must also look for wavelets of lesser dispersion. It is true that the Daubechies wavelets that have finite supports can be adapted to a non-dyadic analysis. Nevertheless, we choose to look elsewhere for our wavelets. Our recourse will be to derive the wavelets from energy functions specified in the frequency domain. By increasing the dispersion of these frequency-domain functions, we succeed in decreasing the dispersion of the corresponding wavelets in the time domain.

Much of what transpires in this paper may be regarded as an attempt to preserve the salient properties of the Shannon wavelets while reducing their dispersion in the time domain. In particular, we shall endeavour to maintain the conditions of sequential orthogonality between wavelets in the same band that are manifest amongst the Shannon wavelets. We shall also preserve the symmetry of the wavelets. The cost of doing so is that we must forego the conditions of orthogonality between wavelets in adjacent bands. However, the mutual orthogonality between wavelets in non-adjacent bands will be preserved. The latter conditions are appropriate to the analysis of spectral structures that are separated by intervening dead spaces. The seasonal structures within the airline passenger data, revealed by Figure 6 , provide a case in point.

Before embarking on our own endeavours, we should make some reference to related work. First, it should be acknowledged that a considerable amount of work has been done already in pursuit of a non-dyadic wavelet analysis. The objective can be described as that of partitioning the timefrequency plane in ways that differ from that of the standard dyadic analysis, represented in Figure 1, and of generating the wavelets to accompany the various schemes.

A program for generalising the standard dyadic analysis has led to the so-called wavelet packet analysis, of which Wickerhauser (1994) is one of the principal exponents. An extensive account has also been provided by Percival and Walden (2000). The essential aim, at the outset, is to decompose the frequency interval $[0, \pi]$ into $2^{j}$ equal intervals. Thereafter, a rich variety of strategies are available.

An alternative approach has been developed under the rubric of $M$-band wavelet analysis. This uses a particular type of filter bank architecture to create $M$ equal subdivisions of each of the octave bands of a dyadic analysis. Seminal contributions have been made by Gopinath and Burrus (1992) and by Steppen, Heller, Gopinath and Burrus (1993). The work of Vaidyanathan 
(1990), (1993) on filter banks has also been influential in this connection.

Next, there is the matter of the uses of wavelets in statistical analysis. Here, the developments have been far too diverse and extensive for us to give a reasonable list of citations. However, it is appropriate to draw attention to a special issue of the Philosophical Transactions of the Royal Society of London that has been devoted to the area. Amongst other pieces, it contains an article by Ramsey (1999), which deals with application of wavelets to financial matters, and a survey by Nason and von Sachs (1999), which covers a wide range of statistical issues.

\section{The Shannon Wavelets}

The Shannon wavelet, which is also known as the sinc function, arises from an attempt to derive a time-localised function from an ordinary trigonometrical function. It is the result of applying a hyperbolic taper to the sine wave to give

$$
\operatorname{sinc}(\omega t)=\frac{\sin (\omega t)}{\pi t}
$$

Woodward (1953) was responsible for naming the sinc function. It has been called the Shannon function in recognition of its central role in the Shannon-Nyquist sampling theory - see, for example, Shannon and Weaver (1964) or Boggess and Narcowich (2001).

The Figures 7-9 plot the functions

$$
\begin{aligned}
\phi_{(0)}(t) & =\frac{\sin (\pi t)}{\pi t}, \\
\phi_{(1)}(t) & =\frac{\sin (\pi t / 2)}{\pi t}, \\
\psi_{(1)}(t) & =\frac{\cos (\pi t) \sin (\pi t / 2)}{\pi t},
\end{aligned}
$$

both for $t \in \mathcal{R}$, which is the real line, and for $t \in \mathcal{I}=\{0, \pm 1, \pm 2, \ldots\}$, which is the set of integers representing the points at which the data are sampled. Here, $\phi_{(0)}(t)$ is the fundamental scaling function, whereas $\phi_{(1)}(t)$ is the scaling function at level 1 and $\psi_{(1)}(t)$ is the level- 1 wavelet.

These time-domain functions with $t \in \mathcal{R}$ are the Fourier transforms of the following square-wave or boxcar functions defined in the frequency 
domain:

$$
\begin{aligned}
& \phi_{(0)}(\omega)= \begin{cases}1, & \text { if }|\omega| \in(0, \pi) ; \\
1 / 2, & \text { if } \omega= \pm \pi, \\
0, & \text { otherwise }\end{cases} \\
& \phi_{(1)}(\omega)= \begin{cases}1, & \text { if }|\omega| \in(0, \pi / 2) ; \\
1 / \sqrt{2}, & \text { if } \omega= \pm \pi / 2, \\
0, & \text { otherwise }\end{cases} \\
& \psi_{(1)}(\omega)= \begin{cases}1, & \text { if }|\omega| \in(\pi / 2, \pi) ; \\
1 / \sqrt{2}, & \text { if } \omega= \pm \pi / 2, \\
1 / 2, & \text { if } \omega= \pm \pi, \\
0, & \text { otherwise }\end{cases}
\end{aligned}
$$

Here and elsewhere, we are using the same symbols to denote the timedomain functions and the frequency-domain functions that are their Fourier transforms. The arguments of the functions alone will serve to make the distinctions.

Within the frequency interval $[-\pi, \pi]$ on the real line, the points $\pm \pi$ and $\pm \pi / 2$ constitute a set of measure zero. Therefore, any finite values can be attributed to the ordinates of the functions at these points without affecting the values of their transforms, which are the functions of (2). It is when the frequency-domain functions are sampled at a finite set of points, including the points in question, that it becomes crucial to adhere to the precise specifications of (3).

When $t \in \mathcal{I}$, the time-domain functions of (2) become sequences that correspond to periodic functions in the frequency domain, with a period of $2 \pi$ radians. These functions are derived by superimposing copies of the aperiodic functions of (3) displaced successively by $2 \pi$ radians in both positive and negative directions. Thus, for example, the periodic function derived from $\phi_{(0)}(\omega)$ is

$$
\tilde{\phi}_{(0)}(\omega)=\sum_{j=-\infty}^{\infty} \phi_{(0)}(\omega+2 \pi j) .
$$

which is just a constant function with a value of unity.

We are defining the periodic functions in terms of the closed intervals $[(2 j-1) \pi,(2 j+1) \pi] ; j \in \mathcal{I}$, such that adjacent intervals have a common endpoint. This is subject to the proviso that only half the value of the ordinate at the common endpoint is attributed to each interval. An alternative recourse, to which we resort elsewhere, is to define the periodic functions 


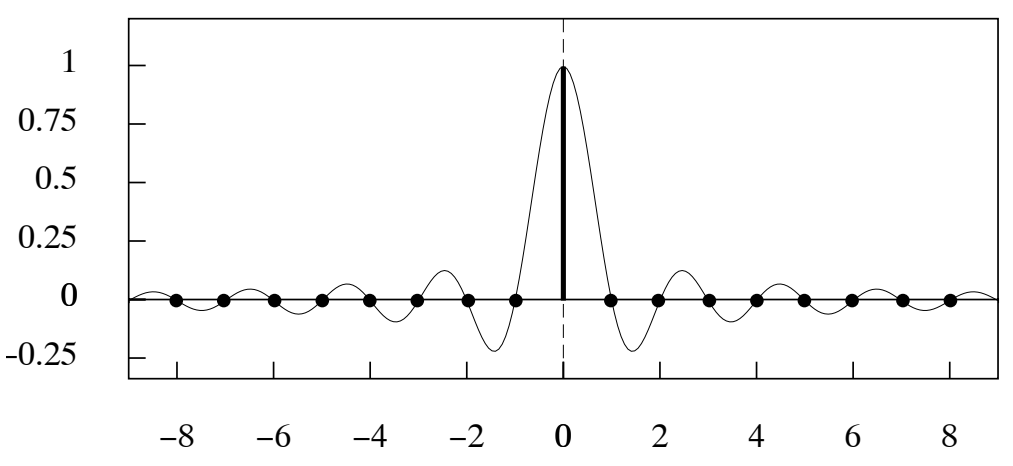

Figure $\%$ The scaling function $\phi_{(0)}(t)$.

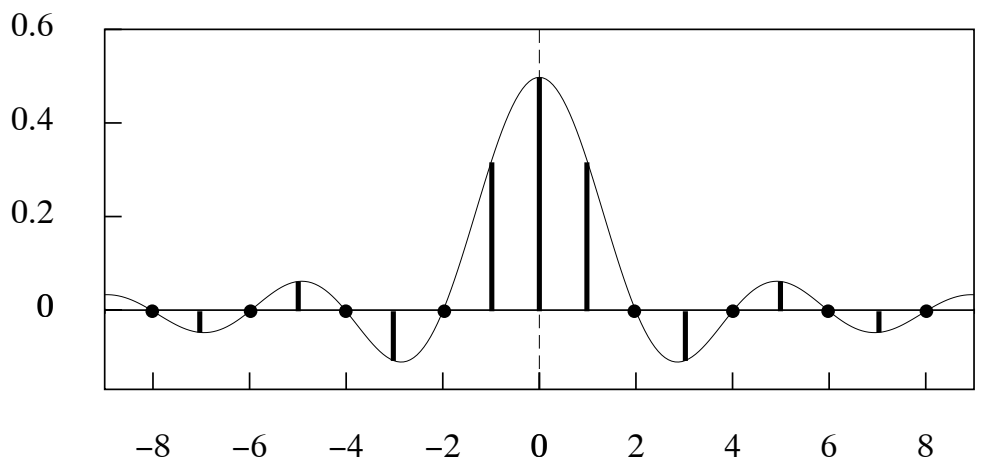

Figure 8. The scaling function $\phi_{(1)}(t)=\phi_{(0)}(t / 2)$.

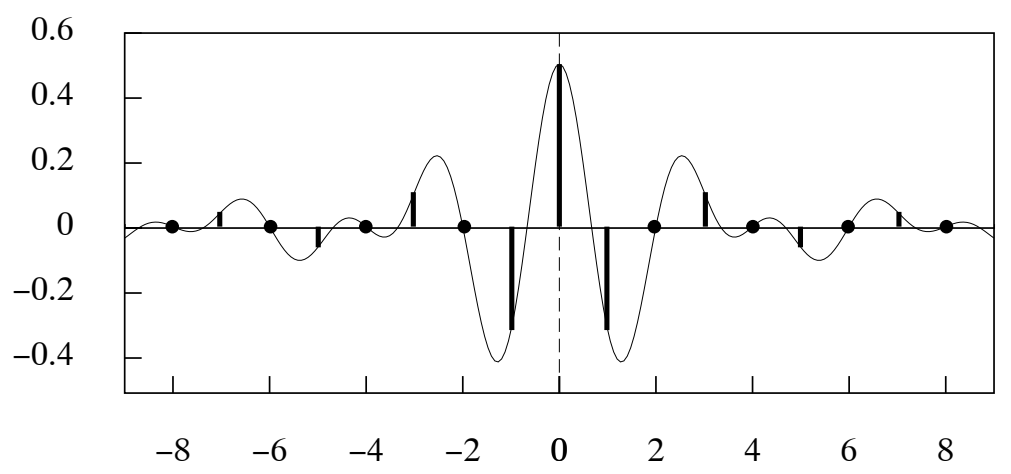

Figure 9. The wavelet function $\psi_{(1)}(t)=\cos (\pi t) \phi_{(0)}(t / 2)$. 
in terms of the non-overlapping half-open intervals such as $[2 \pi[j-1], 2 \pi j)$ and to attribute to the included endpoint the full value of its ordinate.

The time-domain sequences also constitute the coefficients of the ideal frequency-selective filters of which the above-mentioned periodic functions constitute the frequency responses. Given that the frequency responses are real-valued in consequence of the symmetry of the time-domain sequences, they can also be described as the amplitude responses or the gain functions of the filters. In the case of the Shannon wavelet, the periodic frequency functions also represent the energy spectra of the wavelets.

The fundamental scaling function $\phi_{(0)}(t)$ with $t \in \mathcal{I}$, which is depicted in Figure 7, is nothing but the unit impulse sequence. Therefore, the set of sequences $\left\{\phi_{(0)}(t-k) ; t, k \in \mathcal{I}\right\}$, obtained by integer displacements $k$ of $\phi_{(0)}(t)$, constitute the ordinary Cartesian basis in the time domain for the set of all real-valued time series.

The level-1 scaling function $\phi_{(1)}(t)=\phi_{(0)}(t / 2)$ of Figure 8 is derived from the level 0 function by a dilation that entails doubling its temporal dispersion. The level 1 wavelet function $\psi_{(1)}(t)$ of Figure 9 is derived from $\phi_{(1)}(t)$ by a process of frequency shifting, which involves multiplying the latter by $\cos (\pi t)$, which is $(-1)^{t}$ when $t \in \mathcal{I}$, which carries the function into the upper half of the frequency range.

The set of displaced scaling sequences $\left\{\phi_{(1)}(t-2 k) ; t, k \in \mathcal{I}\right\}$, which are separated from each other by multiples of two points, provides a basis for the space of all sequences that are band limited to the frequency range $(0, \pi / 2)$. The corresponding set of wavelet sequences $\left\{\psi_{(1)}(t-2 k) ; t, k \in\right.$ $\mathcal{I}$ \}, which is, in effect, a version of the scaling set that has undergone a frequency translation, provides a basis for the upper frequency range $(\pi / 2, \pi)$. From the fact that, with the exclusion of the boundary points, the two ranges are non-overlapping, it follows that the two basis sets are mutually orthogonal (since sinusoids at different frequencies are mutually orthogonal.) Therefore, the two sets together span the full range $(0, \pi)$.

The elements within the basis sets are also mutually orthogonal. To see this, consider the fact that the boxcar frequency-response functions are idempotent. When multiplied by themselves they do not change, albeit that, with the resulting change of units, they come to represent the energy spectra of the wavelets. The time-domain operation corresponding to this frequency-domain multiplication is autoconvolution. The time-domain functions are real and symmetric, so their autoconvolution is the same as their autocorrelation. Therefore, the discrete wavelet sequences are their own autocorrelation functions. (We should say that, in this context, we are talking of autocorrelations where, in strict parlance, a statistician might talk of autocovariances.)

On inspecting the graphs of these functions, we see that there are zeros 
at the points indexed by $k=2 t$, which correspond to the conditions of orthogonality. We may describe the mutual orthogonality of the displaced wavelets as sequential orthogonality. Orthogonality conditions that extend across frequency bands may be described as lateral orthogonality.

To represent these relationships algebraically, we may consider a wavelet and its transform denoted by $\psi(t) \longleftrightarrow \psi(\omega)$. The autoconvolution of the wavelet gives the autocorrelation function $\xi^{\psi}(t)=\psi(t) * \psi(-t)=\psi(t) *$ $\psi(t)$, where the second equality is in consequence of the symmetry of the wavelet. The corresponding operation in the frequency domain gives the modulation product $\xi^{\psi}(\omega)=\psi(\omega) \psi(-\omega)=\{\psi(\omega)\}^{2}$, where the second equality is in consequence of the fact that the Fourier transform of a realvalued symmetric sequence is also real-valued and symmetric. Thus, there is

$$
\xi^{\psi}(t)=\psi(t) * \psi(t) \longleftrightarrow \xi^{\psi}(\omega)=\{\psi(\omega)\}^{2},
$$

where $\xi^{\psi}(\omega)$ is the energy spectrum of the wavelet. The peculiar feature of the Shannon wavelet is that $\psi(t)=\xi^{\psi}(t)$, for all $t$. The corresponding boxcar function has $\psi(\omega)=\xi^{\psi}(\omega)$, everywhere except at the points of discontinuity.

The conventional dyadic multiresolution wavelet analysis, represented by Figure 1, is concerned with a succession of frequency intervals in the form of $\left(\pi / 2^{j}, \pi / 2^{j-1}\right) ; j=1,2, \ldots, n$, of which the bandwidths are halved repeatedly in the descent from high frequencies to low frequencies. By the $j$ th round, there will be $j$ wavelet bands and one accompanying scalingfunction band.

By applying the scheme described by Mallat (1989), known as the pyramid algorithm, to the discrete versions of the functions, $\phi_{(1)}(t)$ and $\psi_{(1)}(t)$, sets of wavelet sequences can be generated that span these bands. The generic set at level $j$, denoted by $\left\{\psi_{(j)}\left(t-2^{j} k\right) ; t, k \in \mathcal{I}\right\}$, contains mutually orthogonal sequences that are separated by multiples of $2^{j}$ points, and it is accompanied by a set of scaling sequences $\left\{\phi_{(j)}\left(t-2^{j} k\right) ; t, k \in \mathcal{I}\right\}$ that span the lower frequency band $\left[0, \pi / 2^{j}\right)$. (Here, as before, $t$ is the index of the sequence, whereas $k$ is the index of its displacement relative to the other wavelet sequences within the same band.)

A dyadic wave-packet analysis extends this scheme so that, by the $j$ th round, there are $2^{j}$ bands of equal width spanning the intervals ([ $\ell-$ $\left.1] \pi / 2^{j}, \ell \pi / 2^{j}\right) ; \ell=1, \ldots, 2^{j}$. Each such band is spanned by a set of orthogonal functions $\left\{\psi_{\left(\ell / 2^{j}\right)}\left(t-2^{j} k\right) ; t, k \in \mathcal{I}\right\}$ which are separated by multiples of $2^{j}$ points. The first and the second of these bands - counting in terms of rising frequencies, which reverses the dyadic convention-are spanned by the functions $\left\{\psi_{\left(1 / 2^{j}\right)}\left(t-2^{j} k\right)=\phi_{(j)}\left(t-2^{j} k\right)\right\}$ and $\left\{\psi_{\left(2 / 2^{j}\right)}(t-\right.$ 


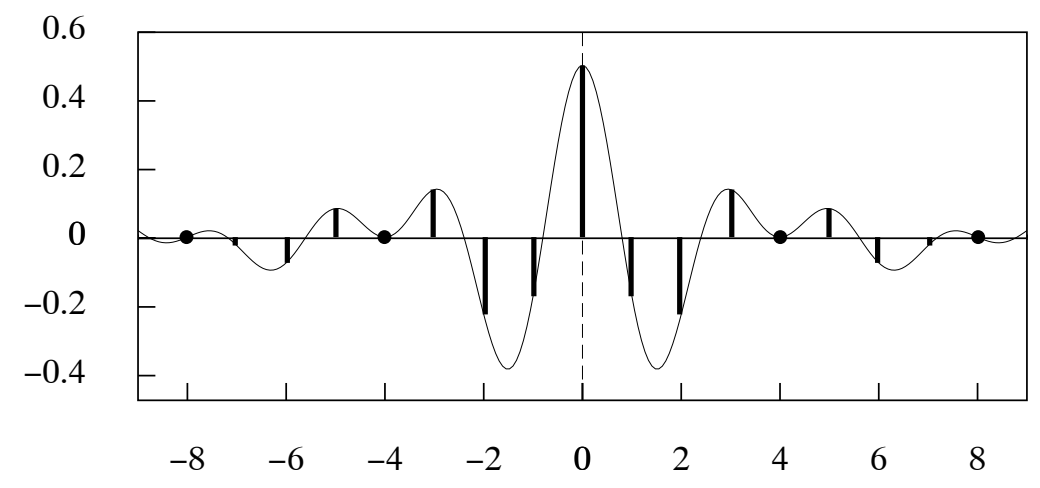

Figure 10. A wavelet within a frequency band of width $\pi / 2$ running from $3 \pi / 8$ to $7 \pi / 8$.

$\left.\left.2^{j} k\right)=\psi_{(j)}\left(t-2^{j} k\right)\right\}$ respectively, which are also found in the dyadic multiresolution wavelet analysis.

In order to generalise such schemes, we need to consider dividing the frequency range by other prime numbers and their products. For this purpose, we must consider the function defined in the frequency domain by

$$
\psi(\omega)= \begin{cases}1, & \text { if }|\omega| \in(\alpha, \beta) \\ 1 / \sqrt{2}, & \text { if } \omega= \pm \alpha, \pm \beta \\ 0, & \text { otherwise }\end{cases}
$$

In case it is required to divide the range into $p$ equal intervals, there will be $\alpha=\pi(j-1) / p$ and $\beta=\pi j / p ; j=1, \ldots, p$. The corresponding time-domain function is

$$
\begin{aligned}
\psi(t)=\frac{1}{\pi t}\{\sin (\beta t)-\sin (\alpha t)\} & =\frac{2}{\pi t} \cos \{(\alpha+\beta) t / 2\} \sin \{(\beta-\alpha) t / 2\} \\
& =\frac{2}{\pi t} \cos (\gamma t) \sin (\delta t),
\end{aligned}
$$

where $\gamma=(\alpha+\beta) / 2$ is the centre of the pass band and $\delta=(\beta-\alpha) / 2$ is half its width. The equality, which follows from the identity $\sin (A+B)-$ $\sin (A-B)=2 \cos A \sin B$, suggests two interpretations. On the LHS is the difference between the coefficients of two lowpass filters with cut-off frequencies of $\beta$ and $\alpha$ respectively. On the RHS is the result of shifting a lowpass filter with a cut-off frequency of $\delta$ so that its centre is moved from $\omega=0$ to $\omega=\gamma$.

The process of frequency shifting is best understood by taking account of both positive and negative frequencies when considering the lowpass filter. Then, the pass band covers the interval $(-\delta, \delta)$. To convert to the bandpass filter, two copies of the pass band are made that are shifted so that their 
new centres lie at $-\gamma$ and $\gamma$. The pass bands have twice the width that one might expect. In the limiting case, the copies are shifted to the centres $-\pi$ and $\pi$. There they coincide, and we have $\psi(t)=2 \cos (\pi t) \sin (\delta t) / \pi t$. To reconcile this with formula for $\psi_{(1)}(t)$ of $(2)$, wherein $\delta=\pi / 2$, we must divide by 2 .

We shall show, in Section 7 , that, when the interval $[0, \pi]$ is partitioned by a sequence of $p$ frequency bands of equal width, an orthogonal basis can be obtained for each band by displacing its wavelets successively by $p$ elements at a time. We shall also show that, when such a band of width $\pi / p$ is shifted in frequency by an arbitrary amount, the conditions of orthogonality will be maintained amongst wavelets that are separated by $2 p$ elements.

This fact, which does not appear to have been recognised previously, can be exploited in fitting pass bands around localised frequency structures that do not fall within the divisions of an even grid. For the present, we shall do no more than illustrate the fact with Figure 10, which shows the effect of translating the Shannon scaling function $\phi_{(1)}(t)$ of width $\pi / 2$ up the frequency scale by an arbitrary amount. It can be see that there are orthogonality conditions affecting wavelets at displacements that are multiples of 4 points.

\section{Compound Filters}

The algorithms of wavelet analysis owe their efficiency to the manner in which the various bandpass filters can be constructed from elementary component filters. The resulting filters may be described as compound filters. The manner in which the filters are formed is expressed more readily in the frequency domain than in the time domain. The subsequent translation of the compound filters from the frequency domain to the time domain is straightforward.

Figure 11 represents, in graphical terms, the construction of the secondlevel scaling function $\phi(2 \omega) \phi(\omega)$ and wavelet $\psi(2 \omega) \phi(\omega)$. These are shown in the third row of the diagram. The fourth row of the diagram shows the remaining wave-packet functions, which come from dividing the domain of the (level-1) wavelet $\psi(\omega)$ in half. The functions, which are defined over the real line, have a period of $2 \pi$. Therefore, they extend beyond the interval $[-\pi, \pi]$ which covers only the central segment. The serrated edges in the diagram are to indicate the severance of the segment from the rest of the function.

To represent the construction algebraically, we may use $\psi_{j / N}(\omega)$ to denote the $j$ th filter in a sequence of $N$ filters that divide the frequency range into equal bands, running from low frequency to high frequency. Then, the 

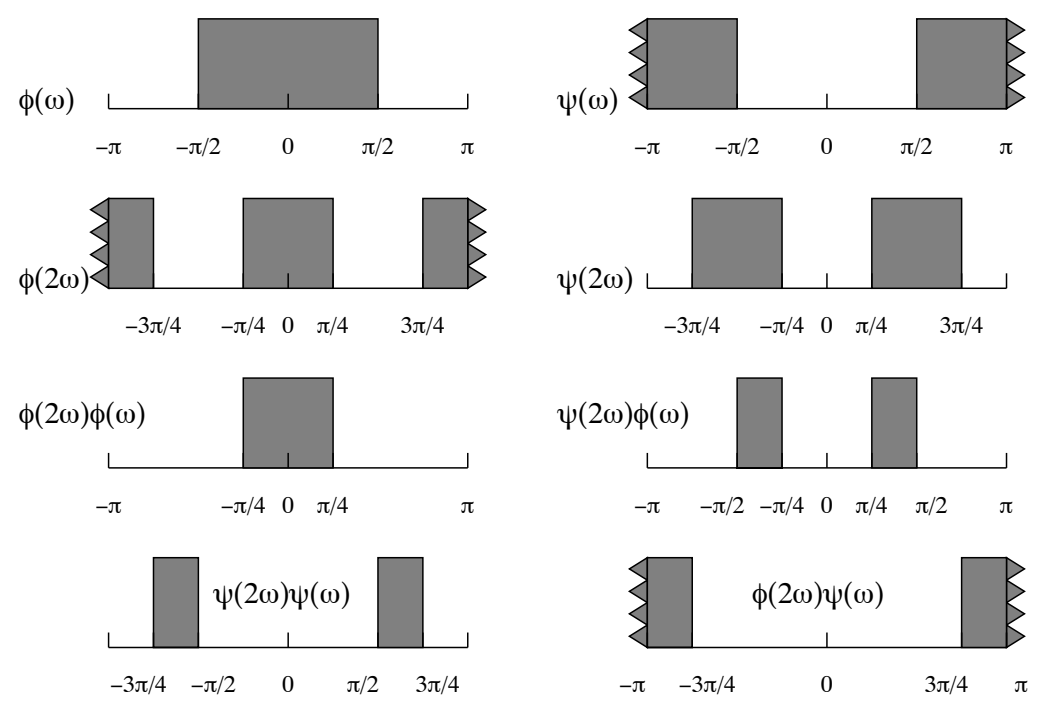

Figure 11. The formation of second-level wavelets and scaling functions illustrated in terms of their frequency-response functions.

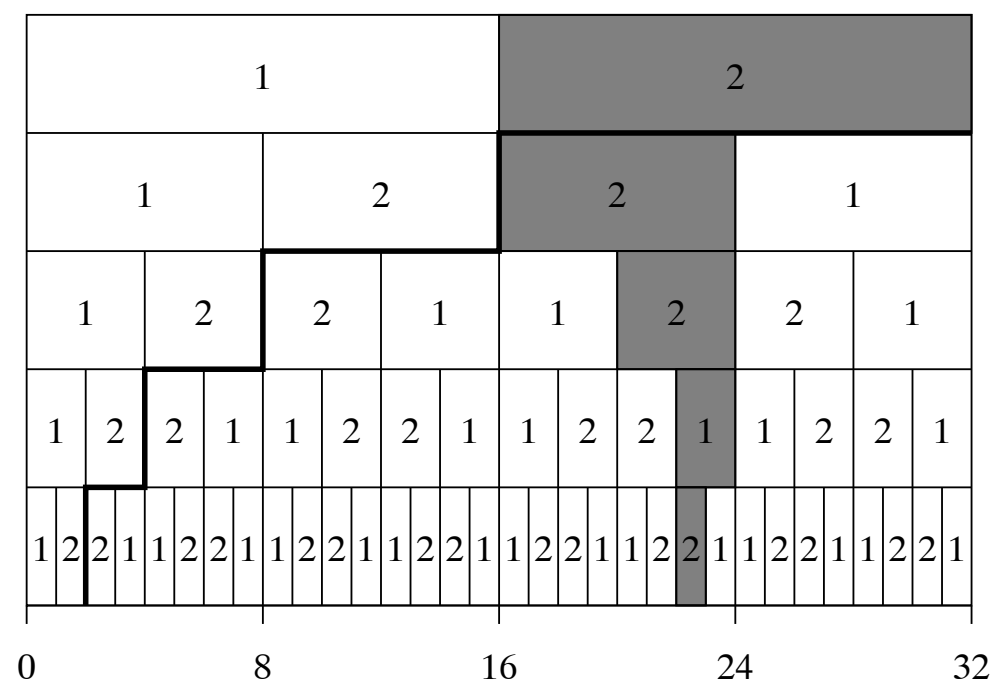

Figure 12. The scheme for constructing compound filters in the dyadic case. The diagram highlights the construction of the filter $\psi_{23 / 32}(\omega)$. 
level-1 scaling function is $\phi_{(1)}(\omega)=\psi_{1 / 2}(\omega)$ and the level- 1 wavelet function is $\psi_{(1)}(\omega)=\psi_{2 / 2}(\omega)$. The second-level scaling function is $\phi_{(2)}(\omega)=\phi_{1 / 4}(\omega)$, whereas the second-level wavelet is $\psi_{(2)}(\omega)=\psi_{2 / 4}(\omega)$. The algebra for the second-level functions is as follows:

$$
\begin{aligned}
\phi_{(2)}(\omega)= & \phi_{1 / 4}(\omega)=\phi_{(1)}(2 \omega) \phi_{(1)}(\omega)=\psi_{1 / 2}(2 \omega) \psi_{1 / 2}(\omega), \\
\psi_{(2)}(\omega)= & \psi_{2 / 4}(\omega)=\psi_{(1)}(2 \omega) \phi_{(1)}(\omega)=\psi_{2 / 2}(2 \omega) \psi_{1 / 2}(\omega), \\
& \psi_{3 / 4}(\omega)=\psi_{(1)}(2 \omega) \psi_{(1)}(\omega)=\psi_{2 / 2}(2 \omega) \psi_{2 / 2}(\omega), \\
\psi_{4 / 4}(\omega) & =\phi_{(1)}(2 \omega) \psi_{(1)}(\omega)=\psi_{1 / 2}(2 \omega) \psi_{2 / 2}(\omega) .
\end{aligned}
$$

The formulae for the filters at the $(j+1)$ th level of an ordinary dyadic analysis, of the kind depicted in Figure 1, are

$$
\begin{aligned}
& \phi_{(j+1)}(\omega)=\phi_{(j)}(2 \omega) \phi_{(1)}(\omega)=\phi_{(1)}\left(2^{j} \omega\right) \phi_{(j)}(\omega), \\
& \psi_{(j+1)}(\omega)=\psi_{(j)}(2 \omega) \phi_{(1)}(\omega)=\psi_{(1)}\left(2^{j} \omega\right) \phi_{(j)}(\omega) .
\end{aligned}
$$

The equalities can be established via recursive expansions of the formulae. Regardless of which of the forms are taken, we get

$$
\phi_{(j+1)}(\omega)=\prod_{i=0}^{j} \phi_{(1)}\left(2^{i} \omega\right) \quad \text { and } \quad \psi_{(j+1)}(\omega)=\psi_{(1)}(\omega) \prod_{i=1}^{j} \phi_{(1)}\left(2^{i} \omega\right) .
$$

The formulae of (9) can be translated into the time domain. A modulation in the frequency domain corresponds to a convolution in the time domain. Raising the frequency value of any function $\psi_{(k)}(\omega)$ by a factor of $n$ entails interpolating $n-1$ zeros between the elements of the corresponding time-domain sequence $\psi_{(k)}(t)$ to give a sequence that may be denoted by $\psi_{(k)}(t \uparrow n)$. Thus, it can be seen that

$$
\begin{aligned}
& \phi_{(j+1)}(t)=\phi_{(j)}(t \uparrow 2) * \phi_{(1)}(t)=\phi_{(1)}\left(t \uparrow 2^{j}\right) * \phi_{(j)}(t), \\
& \psi_{(j+1)}(t)=\psi_{(j)}(t \uparrow 2) * \phi_{(1)}(t)=\psi_{(1)}\left(t \uparrow 2^{j}\right) * \phi_{(j)}(t) .
\end{aligned}
$$

As they stand, these time-domain formulae are not practical: the sequences $\phi_{(1)}(t)$ and $\psi_{(1)}(t)$ of the ordinates of the Shannon functions are infinite and they converge none too rapidly. The practical finite-sample versions of the formulae will be derived in the next section.

Figure 12 shows how the dyadic scheme for forming compound filters can be extended through successive rounds; and it portrays the subdivision of the wavelets bands to create a set of bands of equal width that cover the entire frequency range. The figure represents five successive rounds; and it highlights the construction of the bandpass filter which is the 23rd in 
a succession of 32 filters with pass bands of ascending frequency. In these terms, the filter is

$$
\psi_{23 / 32}(\omega)=\psi_{2 / 2}(16 \omega) \psi_{1 / 2}(8 \omega) \psi_{2 / 2}(4 \omega) \psi_{2 / 2}(2 \omega) \psi_{2 / 2}(\omega) .
$$

The bold lines in Figure 12, which create a flight of steps descending from right to left, relate to the pyramid algorithm of the ordinary dyadic multiresolution analysis. In the $j$ th round, the algorithm separates into two components a filtered sequence that is associated with frequency interval $\left(0, \pi / 2^{j-1}\right)$. From the high-frequency component are derived the amplitude coefficients of the wavelets of the $j$ th level. The low-frequency component is passed to the next round for further subdivision.

To see how the dyadic scheme may be generalised, consider the case where the positive frequency range $[0, \pi]$ is already divided into $n$ equal intervals, by virtue of $n$ bandpass filters denoted $\psi_{1 / n}(\omega), \ldots, \psi_{n / n}(\omega)$. The objective is to subdivide each interval into $p$ sub intervals, where $p$ is a prime number.

Imagine that there also exists a set of $p$ bandpass filters, $\psi_{1 / p}(\omega)$, $\ldots, \psi_{p / p}(\omega)$, that partition the interval $[0, \pi]$ into $p$ equal parts. Amongst the latter, the ideal specification of the generic bandpass filter is

$$
\psi_{j / p}(\omega)= \begin{cases}1, & \text { if }|\omega| \in \mathcal{I}_{j}, \\ 1 / 2 & \text { if }|\omega|=(j-1) \pi / p, j \pi / p, \\ 0, & \text { if }|\omega| \in \mathcal{I}_{j}^{c},\end{cases}
$$

where the open interval $\mathcal{I}_{j}=([j-1] \pi / p, j \pi / p)$ is the $j$ th of the $p$ subdivisions of $[0, \pi]$, and where $\mathcal{I}_{j}^{c}$ is the complement within $[0, \pi]$ of the closed interval $\mathcal{I}_{j} \cup\{(j-1) \pi / p, j \pi / p\}$ that includes the endpoints. But the function $\psi_{j / p}(\omega)$ is symmetric such that $\psi_{j / p}(\omega-\pi)=\psi_{j / p}(\pi-\omega)$. It also has a period of $2 \pi$ such that $\psi_{j / p}(\omega-\pi)=\psi_{j / p}(\omega+\pi)$. The two conditions imply that $\psi_{j / p}(\pi+\omega)=\psi_{j / p}(\pi-\omega)$. It follows that

$$
\psi_{j / p}(\pi+\omega)= \begin{cases}1, & \text { if }|\omega| \in \mathcal{I}_{p+1-j}, \\ 0, & \text { if }|\omega| \in \mathcal{I}_{p+1-j}^{c},\end{cases}
$$

where $\mathcal{I}_{p+1-j}^{c}$ in the $j$ th interval in the reverse sequence $\left\{\mathcal{I}_{p}, \mathcal{I}_{p-1}, \ldots, \mathcal{I}_{1}\right\}$.

To subdivide the first of the $n$ intervals, which is $(0, \pi / n)$, into $p$ parts, the filters $\psi_{1 / p}(n \omega), \ldots, \psi_{p / p}(n \omega)$ are used, in which the argument $\omega$ has been multiplied by $n$. These have the same effect on the first interval as the original filters have on the interval $[0, \pi]$. To subdivide the second of the $n$ intervals, which is $(\pi / n, 2 \pi / n)$, the filters $\psi_{p / p}(n \omega), \ldots, \psi_{1 / p}(n \omega)$ are used, which are in reversed order. For, in this case, $\omega \in(\pi / n, 2 \pi / n)$ gives $n \omega=\pi+\lambda$ with $\lambda \in(0, \pi)$; and, therefore, the conditions of (14) apply. 


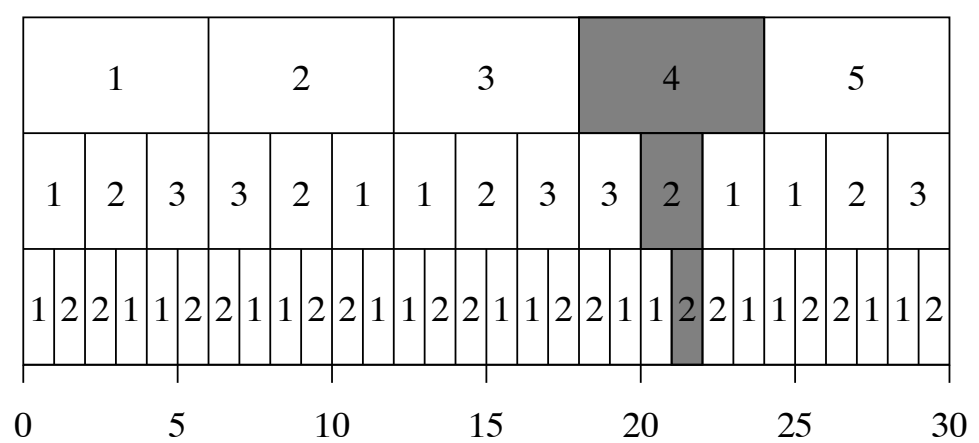

Figure 13. The 22nd bandpass filter out of 30 factorised as $\psi_{22 / 30}(\omega)=\psi_{2 / 2}(15 \omega) \psi_{2 / 3}(5 \omega) \psi_{4 / 5}(\omega)$.

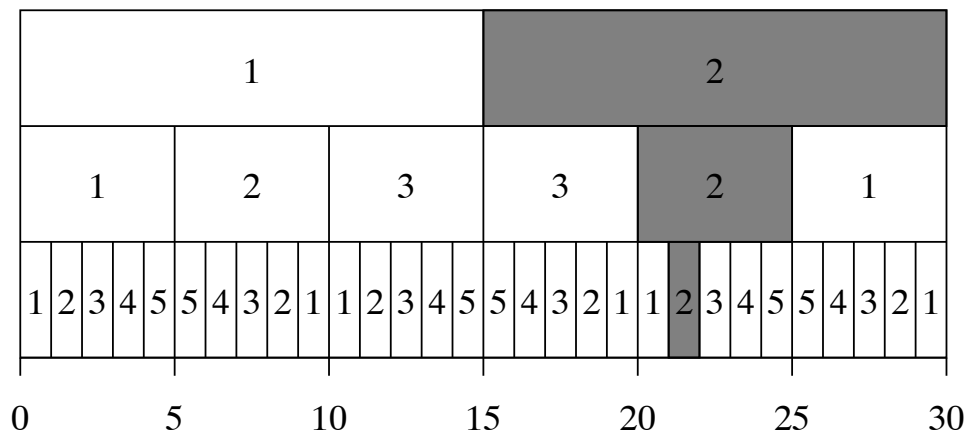

Figure 14. The 22nd bandpass filter out of 30 factorised as $\psi_{22 / 30}(\omega)=\psi_{2 / 5}(6 \omega) \psi_{2 / 3}(2 \omega) \psi_{2 / 2}(\omega)$.

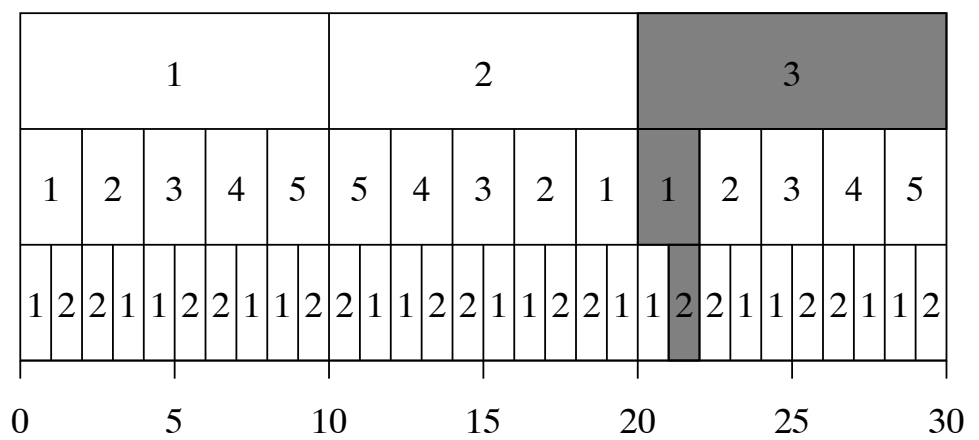

Figure 15. The 22nd bandpass filter out of 30 factorised as $\psi_{22 / 30}(\omega)=\psi_{2 / 2}(15 \omega) \psi_{1 / 5}(3 \omega) \psi_{3 / 3}(\omega)$. 
Now we may recognise that the $2 \pi$ periodicity of $\psi_{j / p}(\omega)$ implies that, amongst the $n$ intervals that are to be sub divided, all odd-numbered intervals may be treated in the manner of the first interval, whereas all evennumbered intervals may be treated in the manner of the second interval.

The generic compound filter, which has a pass band on the $j$ th interval out of $n p$ intervals, is specified by

$$
\psi_{j / p n}(\omega)=\psi_{k / p}(n \omega) \psi_{\ell / n}(\omega)
$$

where

$$
\ell=(j \operatorname{div} p)+1 \quad \text { and } \quad k= \begin{cases}(j \bmod p), & \text { if } \ell \text { is odd } \\ p+1-(j \bmod p), & \text { if } \ell \text { is even }\end{cases}
$$

Here, $(j \operatorname{div} p)$ is the quotient of the division of $j$ by $p$ and $(j \bmod p)$ is the remainder. (Reference to the first two rows of Figures 13-15 will help in verifying this formula.)

Given a succession of prime factors, some of which may be repeated, the formula of (15) may be used recursively to construct compound filters of a correspondingly composite nature. However, whereas the prime factorisation of the sample size $T=p_{1} p_{2} \cdots p_{q}$ is unique, the order of the factors is arbitrary. By permuting the order, one can find a variety of compositions that amount to the same bandpass filter.

Figures 13-15 represent three ways of constructing the filter $\psi_{22 / 30}$ from elementary components, which are from the sets $\left\{\psi_{j / 2}, j=1,2\right\}$, $\left\{\psi_{k / 3}, k=1,2,3\right\}$ and $\left\{\psi_{\ell / 5}, \ell=1,2, \ldots, 5\right\}$. There are altogether 6 ways in which the filter may be constructed; but it seems reasonable, to opt for the construction, represented by Figure 13, that takes the prime factors in order of descending magnitude. In practice, the filters are represented, in the frequency domain, by the ordinates of their frequency response functions, sampled at equal intervals; and the ordering of the factors by declining magnitude will serve to minimise the number of multiplications entailed in the process of compounding the filters. This is reflected in the fact that, compared with the other figures, Figure 13 has the least highlighted area.

Raising the frequency value of $\psi_{k / p}(\omega)$ by a factor of $n$ entails interpolating $n-1$ zeros between every point of the corresponding time-domain sequence $\psi_{k / p}(t)$. The following expression indicates the correspondence between the equivalent operations of compounding the filter in the time domain and the frequency domain:

$$
\psi_{j / n p}(t)=\left\{\psi_{k / p}(t) \uparrow n\right\} * \psi_{\ell / n}(t) \longleftrightarrow \psi_{j / n p}(\omega)=\psi_{k / p}(n \omega) \psi_{\ell / n}(\omega) .
$$

When creating a compound filter via convolutions in the time domain, the prime factors should be taken in ascending order of magnitude. 
In this section, we have described a method of generating the wavelets by compounding a series of filters. In theory, the process can be pursued either in the time domain, via convolutions, or in the frequency domain, via modulations. In the case of the Shannon wavelets, the frequency domain specifications at all levels use the same rectangular template, which is mapped onto the appropriate frequency intervals. Therefore, is makes no difference whether the wavelets are produced via the compounding process or directly from the template, appropriately scaled and located in frequency.

For other wavelet specifications, a choice must be made. Either they are generated by a process of compounding, which is generally pursued in the time domain using a fixed set of dilation coefficients as the template, or else they are generated in the frequency domain using a fixed energy-function template. The results of the two choices may be quite different. It is the latter choice that we shall make in the remainder of this paper.

Example. The Daubechies D4 wavelet and the scaling function of Figures 2 and 3 relate to a dyadic analysis that proceeds in the time domain on the basis of a set of four dilation coefficients. The dilation coefficients for the scaling function are $p_{0}=(1+\sqrt{3}) / 4, p_{1}=(3+\sqrt{3}) / 4, p_{2}=(3-\sqrt{3}) / 4$ and $p_{3}=(\sqrt{3}-1) / 4$. The coefficients that are used in creating the wavelets from the scaling functions are $q_{0}=p_{3}, q_{1}=-p_{3}, q_{2}=p_{1}$ and $q_{3}=-p_{0}$. The sequences $p_{(1)}(t)=\left\{p_{t}\right\}$ and $q_{(1)}(t)=\left\{q_{t}\right\}$ may be compared to the sequences of Shannon coefficients $\phi_{(1)}(t)$ and $\psi_{(1)}(t)$ respectively. On that basis, the following equations can be defined, which correspond to those of (11):

$$
\begin{aligned}
& p_{(j+1)}(t)=p_{(j)}(t \uparrow 2) * p_{(1)}(t)=p_{(1)}\left(t \uparrow 2^{j}\right) * p_{(j)}(t), \\
& q_{(j+1)}(t)=p_{(j)}(t \uparrow 2) * q_{(1)}(t)=q_{(1)}\left(t \uparrow 2^{j}\right) * p_{(j)}(t) .
\end{aligned}
$$

The first of the alternative forms, which entails the interpolation of a zero between each of the coefficients of $p_{(j)}(t)$, corresponds to the recursive system that has been used in generating Figures 2 and 3 . That is to say, the diagrams have been created by proceeding through a number of iterations and then mapping the resulting coefficients, which number $2^{j+1}+2^{j}-2$ at the $j$ th iteration, into the interval $[0,3]$. The difference between the wavelets and the scaling functions lies solely in the starting values. This algorithm provides a way of seeking the fixed-point solution to the following dilation equation that defines the D4 scaling function $\phi_{D}(t)$ with $t \in \mathcal{R}$ :

$$
\phi_{D}(t)=p_{0} \phi_{D}(2 t)+p_{1} \phi_{D}(2 t-1)+p_{2} \phi_{D}(2 t-2)+p_{3} \phi_{D}(2 t-3) .
$$

The second of the forms is implicated in the pyramid algorithm of Mallat (1989). In this case, the difference between the wavelets and the scaling functions lies solely in the final iteration. 


\section{Adapting to Finite Samples}

The wavelet sequences corresponding to the ideal bandpass filters are defined on the entire set of integers $\{t=0, \pm 1, \pm 2, \ldots\}$ whereas, in practice, a discrete wavelet analysis concerns a sample of $T$ data points. This disparity can be overcome, in theory, by creating a periodic extension of the data that replicates the sample in all intervals of the duration $T$ that precede and follow it. By this means, the data value at a point $t \notin\{0,1, \ldots, T-1\}$, which lies outside the sample, is provided by $y_{t}=y_{\{t \bmod T\}}$, where $(t \bmod T)$ lies within the sample. With the periodic extension available, one can think of multiplying the filter coefficients point by point with the data.

As an alternative to extending the data, one can think of creating a finite sequence of filter coefficients by wrapping the infinite sequence $\psi(t)=\left\{\psi_{t}\right\}$ around a circle of circumference $T$ and adding the overlying coefficients to give

$$
\psi_{t}^{\circ}=\sum_{k=-\infty}^{\infty} \psi_{\{t+k T\}} \quad \text { for } \quad t=0,1, \ldots, T-1
$$

The inner product of the resulting coefficients $\psi_{0}^{\circ}, \ldots, \psi_{T-1}^{\circ}$ with the sample points $y_{0}, \ldots, y_{T-1}$ will be identical to that of the original coefficients with the extended data. To show this, let $\tilde{y}(t)=\left\{\tilde{y}_{t}=y_{\{t \bmod T\}}\right\}$

denote the infinite sequence that is the periodic extension of $y_{0}, \ldots, y_{T-1}$. Then,

$$
\begin{aligned}
\sum_{t=-\infty}^{\infty} \psi_{t} \tilde{y}_{t} & =\sum_{k=-\infty}^{\infty}\left\{\sum_{t=0}^{T-1} \psi_{\{t+k T\}} \tilde{y}_{\{t+k T\}}\right\} \\
& =\sum_{t=0}^{T-1} y_{t}\left\{\sum_{k=-\infty}^{\infty} \psi_{\{t+k T\}}\right\}=\sum_{t=0}^{T-1} y_{t} \psi_{t}^{\circ}
\end{aligned}
$$

Here, the first equality, which is the result of cutting the sequence $\left\{\psi_{t} \tilde{y}_{t}\right\}$ into segments of length $T$, is true in any circumstance, whilst the second equality uses the fact that $\tilde{y}_{\{t+k T\}}=y_{\{t \bmod T\}}=y_{t}$. The final equality invokes the definition of $\psi_{t}^{\circ}$.

In fact, the process of wrapping the filter coefficients should be conducted in the frequency domain, where it is simple and efficient, rather than in the time domain, where it entails the summation of infinite series. We shall elucidate these matters while demonstrating the use of the discrete Fourier transform in performing a wavelets analysis. 
To begin, let us consider the $z$-transforms of the filter sequence and the data sequence:

$$
\psi(z)=\sum_{t=-\infty}^{\infty} \psi_{t} z^{t} \quad \text { and } \quad y(z)=\sum_{t=0}^{T-1} y_{t} z^{t} .
$$

Setting $z=\exp \{-\mathrm{i} \omega\}$ in $\psi(z)$ creates a continuous periodic function in the frequency domain of period $2 \pi$, denoted by $\psi(\omega)$, which, by virtue of the discrete-time Fourier transform, corresponds one-to-one with the doubly infinite time-domain sequence of filter coefficients.

Setting $z=z_{j}=\exp \{-\mathrm{i} 2 \pi j / T\} ; j=0,1, \ldots, T-1$, is tantamount to sampling the (piecewise) continuous function $\psi(\omega)$ at $T$ points within the frequency range of $\omega \in[0,2 \pi)$. (Given that the data sample is defined on a set of positive integers, it is appropriate to replace the symmetric interval $[-\pi, \pi]$, considered hitherto, in which the endpoints are associated with half the values of their ordinates, by the positive frequency interval $[0,2 \pi)$, which excludes the endpoint on the right and attributes the full value of the ordinate at zero frequency to the left endpoint.) The powers of $z_{j}$ now form a $T$-periodic sequence, with the result that

$$
\begin{aligned}
\psi\left(z_{j}\right) & =\sum_{t=-\infty}^{\infty} \psi_{t} z_{j}^{t} \\
& =\left\{\sum_{k=-\infty}^{\infty} \psi_{k T}\right\}+\left\{\sum_{k=-\infty}^{\infty} \psi_{(k T+1)}\right\} z_{j}+\cdots+\left\{\sum_{k=-\infty}^{\infty} \psi_{(k T+T-1)}\right\} z_{j}^{T-1} \\
& =\psi_{0}^{\circ}+\psi_{1}^{\circ} z_{j}+\cdots+\psi_{T-1}^{\circ} z_{j}^{T-1}=\psi^{\circ}\left(z_{j}\right) .
\end{aligned}
$$

There is now a one-to-one correspondence, via the discrete Fourier transform, between the values $\psi\left(z_{j}\right) ; j=0,1, \ldots, T-1$, sampled from $\psi(\omega)$ at intervals of $2 \pi / T$, and the coefficients $\psi_{0}^{\circ}, \ldots, \psi_{T-1}^{\circ}$ of the circular wrapping of $\psi(t)$. Setting $z=z_{j}=\exp \{-i 2 \pi j / T\} ; j=0,1, \ldots, T-1$, within $y(z)$ creates the discrete Fourier transform of the data sequence, which is commensurate with the square roots of the ordinates sampled from the energy function.

To elucidate the correspondence between operations in the two domains, we may replace $z$ in the equation of (22) by a circulant matrix $K=\left[e_{1}, \ldots, e_{T-1}, e_{0}\right]$, which is formed from the identity matrix $I_{T}=$ $\left[e_{0}, e_{1}, \ldots, e_{T-1}\right]$ of order $T$ by moving the leading vector to the end of the array. Since $K^{q}=K^{T+q}$, the powers of the matrix form a $T$-periodic sequence, as do the powers of $z=\exp \{-\mathrm{i} 2 \pi j / T\}$. (A full account of the algebra of circulant matrices has been provided by Pollock 2002.) 
The matrix $K$ is amenable to a spectral factorisation of the form $K=$ $\bar{U} D U$, where

$$
\begin{aligned}
U & =T^{-1 / 2} W=T^{-1 / 2}[\exp \{-\mathrm{i} 2 \pi t j / T\} ; t, j=0, \ldots, T-1] \text { and } \\
\bar{U} & =T^{-1 / 2} \bar{W}=T^{-1 / 2}[\exp \{\mathrm{i} 2 \pi t j / T\} ; t, j=0, \ldots, T-1]
\end{aligned}
$$

are unitary matrices such that $U \bar{U}=\bar{U} U=I_{T}$, and where

$$
D=\operatorname{diag}\{1, \exp \{-\mathrm{i} 2 \pi / T\}, \ldots, \exp \{-\mathrm{i} 2 \pi(T-1) / T\}\}
$$

is a diagonal matrix whose elements are the $T$ roots of unity, which are found on the circumference of the unit circle in the complex plane.

Using $K=\bar{U} D U$ in place of $z$ in (22) creates the following circulant matrices:

$$
\Psi^{\circ}=\psi^{\circ}(K)=\bar{U} \psi^{\circ}(D) U \quad \text { and } \quad Y=y(K)=\bar{U} y(D) U .
$$

The multiplication of two circulant matrices generates the circular convolution of their elements. Thus the product

$$
\Psi^{\circ} Y=\left\{\bar{U} \psi^{\circ}(D) U\right\}\{\bar{U} y(D) U\}=\bar{U} \psi^{\circ}(D) y(D) U .
$$

is a matrix in which the leading vector contains the elements of the circular convolution of $\left\{\psi_{0}^{\circ}, \ldots, \psi_{T-1}^{\circ}\right\}$ and $\left\{y_{0}, \ldots, y_{T-1}\right\}$, of which the inner product of (21) is the first element.

The leading vector of $\Psi^{\circ} Y$ can be isolated by postmultiplying this matrix by $e_{0}=[1,0, \ldots, 0]^{\prime}$. But $U e_{0}=T^{-1 / 2} W e_{0}=T^{-1 / 2} h$, where $h=[1,1, \ldots, 1]^{\prime}$ is the summation vector. Therefore,

$$
\Psi^{\circ} Y e_{0}=T^{-1} \bar{W}\left\{\psi^{\circ}(D) y(D) h\right\},
$$

where $\psi^{\circ}(D) y(D) h$ is a vector whose elements are the products of the diagonal elements of $\psi^{\circ}(D)$ and $y(D)$. Equation (28) corresponds to the usual matrix representation of an inverse discrete Fourier transform, which maps a vector from the frequency domain into a vector of the time domain.

Observe that equation (27) also establishes the correspondence between the operation of cyclical convolution in the time domain, represented by the product of the matrices on the LHS, and the operation of modulation in the frequency domain, represented by the pairwise multiplication of the elements of two diagonal matrices. The correspondence can be represented by writing $\Psi^{\circ} Y \longleftrightarrow \psi^{\circ}(D) y(D)$. Using such notation, we can represent the finite-sample version of equation (17) by

$$
\psi_{j / n p}^{\circ}(K)=\psi_{k / p}^{\circ}\left(K^{n}\right) \psi_{\ell / n}^{\circ}(K) \longleftrightarrow \psi_{j / n p}^{\circ}(D)=\psi_{k / p}^{\circ}\left(D^{n}\right) \psi_{\ell / n}^{\circ}(D) .
$$


If $\alpha(z)$ is a polynomial of degree $T-1$ and, if $n$ is a factor of $T$, then $\alpha\left(K^{n}\right)=\bar{U} \alpha\left(D^{n}\right) U$ is a circulant matrix of order $T$ in which there are $T / n$ nonzero bands, with $n-1$ bands of zeros lying between one nonzero band and the next. The generic nonzero coefficient, which is on the $t$ th nonzero subdiagonal band, is $\alpha_{t}^{\circ}=\sum_{j=0}^{T / n} \alpha_{\{t+j n\}}$. The $j$ th diagonal element of the matrix $D^{n}$, which is entailed in the spectral factorisation of $\alpha\left(K^{n}\right)$, takes the values $\exp \{-\mathrm{i} 2 \pi n j / T\} ; j=0,1, \ldots, T-1$. Compared to the corresponding elements of $D$, its frequency values have been increased by a factor of $n$.

In the case of a piecewise continuous energy function $\xi(\omega)=|\psi(\omega)|^{2}$, defined on the interval $[-\pi, \pi]$, one can afford to ignore the endpoints of the interval together with any points of discontinuity within the interval. These constitute a set of measure zero in the context of the remaining frequency values. When such points are taken in the context of a sample of $T$ frequency values, they can no longer be ignored, as the example at the the end of this section indicates.

The method of coping with finite samples via a periodic extension of the data is also a feature of a discrete Fourier analysis. It requires the data to be free of an overall trend. Otherwise, there will be a radical disjunction in passing from the end of one replication of the sample to the begining of the next. Such disjunctions will affect all of the Fourier coefficients. However, the effect upon the coefficients of a wavelet analysis will be limited to the extent that the wavelets are localised in time. A disadvantage of the Shannon wavelets is that they are widely dispersed; and, in the next section, we shall be developing wavelets that are more localised.

Example: The Wrapped Shannon Wavelet. Consider a set of frequencydomain ordinates sampled from a boxcar energy function, defined over the interval $[-\pi, \pi]$, at the points $\omega_{j}=2 \pi j / T ; j=1-T / 2, \ldots, 0, \ldots, T / 2$, where $T$ is even:

$$
\xi_{j}^{\circ}= \begin{cases}1, & \text { if } j \in\{1-d, \ldots, d-1\} \\ 1 / 2, & \text { if } j= \pm d, \\ 0, & \text { otherwise. }\end{cases}
$$

Here, $d<T / 2$ is the index of the point of discontinuity. The (inverse) Fourier transform of these ordinates constitutes the autocorrelation function of the wrapped Shannon wavelet. The transform of the square roots of the ordinates is the wavelet itself.

The $z$-transform of the energy sequence is $\xi^{\circ}(z)=\left\{S^{+}(z)+S^{-}(z)\right\} / 2$, wherein 


$$
\begin{aligned}
& S^{+}(z)=z^{-d}+\cdots+z^{-1}+1+z+\cdots+z^{d} \text { and } \\
& S^{-}(z)=z^{1-d}+\cdots+z^{-1}+1+z+\cdots+z^{d-1} .
\end{aligned}
$$

Setting $z=e^{-\mathrm{i} \omega_{1} t}$, where $\omega_{1}=2 \pi / T$, and using the formula for the partial sum of a geometric progression, gives the following Dirichlet kernels:

$$
S^{+}(t)=\frac{\sin \left\{\omega_{1} t(d+1 / 2)\right\}}{\sin \left(\omega_{1} t / 2\right)}, \quad S^{-}(t)=\frac{\sin \left\{\omega_{1} t(d-1 / 2)\right\}}{\sin \left(\omega_{1} t / 2\right)} .
$$

But $\sin (A+B)+\sin (A-B)=2 \sin A \cos B$, so, with $A=\omega_{1} t d$ and $B=$ $\omega_{1} t / 2$, we have

$$
\xi^{\circ}(t)=\frac{1}{2 T}\left\{S^{+}(t)+S^{-}(t)\right\}=\frac{\cos \left(\omega_{1} t / 2\right) \sin \left(d \omega_{1} t\right)}{T \sin \left(\omega_{1} t / 2\right)},
$$

This expression gives the values of the circular autocorrelation function of the wrapped wavelet at the points $t=1, \ldots, T-1$. The value at $t=0$ is $\xi_{0}^{\circ}=2 d / T$, which comes from setting $z=1$ in the expressions for $S^{+}(z)$ and $S^{-}(z)$ of $(31)$.

If $d=T / 4$, such that the points of discontinuity are at $\pm \pi / 2$, as in the specification of $\phi_{(0)}(t)$ under (3), then $\sin \left(d \omega_{1} t\right)=\sin (\pi t / 2)$ and $\xi^{\circ}(2 t)=0$ for $t=1, \ldots, T-1$. This confirms that the relevant conditions of sequential orthogonality are indeed fulfilled by the wrapped wavelet.

The technique of frequency shifting may be applied to the formula of (33). Let $g$ be the index that marks the centre of the pass band. Then, the autocorrelation function of the wrapped wavelet corresponding to a bandpass filter with lower and upper cut-off points of $a=g-d$ and $b=g+d$ is given by

$$
\xi^{\circ}(t)=2 \cos \left(g \omega_{1} t\right) \frac{\cos \left(\omega_{1} t / 2\right) \sin \left(d \omega_{1} t\right)}{T \sin \left(\omega_{1} t / 2\right)} .
$$

To find the wavelets themselves, we transform a set of frequency-domain coefficients that are the square roots of those of the energy function. For the wavelet corresponding to the ideal lowpass filter with a cut-off at $j= \pm d$, we have

$$
T \phi^{\circ}(t)=\frac{\sin \left\{\omega_{1} t(d-1 / 2)\right\}}{\sin \left(\omega_{1} t / 2\right)}+\sqrt{2} \cos \left(d \omega_{1}\right) .
$$

For the wavelet corresponding to the ideal bandpass filter with a cut-off points at $j= \pm a, \pm b$, there is

$$
\begin{aligned}
T \psi^{\circ}(t)=2 \cos \left\{(a+b) \omega_{1} t / 2\right\} \frac{\sin \left\{(b-a-1) \omega_{1} t / 2\right\}}{\sin \left(\omega_{1} t / 2\right)} \\
+\sqrt{2}\left\{\cos \left(a \omega_{1}\right)+\cos \left(b \omega_{1}\right)\right\} .
\end{aligned}
$$




\section{Conditions of Sequential Orthogonality in the Dyadic Case}

The advantage of the Shannon wavelets is that they provide us with a ready-made orthogonal bases for the frequency bands that accompany a multiresolution analysis or a wave packet analysis. We have illustrated this feature, in Section 3, with the case of the infinite wavelet and scaling function sequences that correspond to the first level of a dyadic analysis.

The conditions of orthogonality also prevail in the case of the wrapped wavelet sequence. This may be demonstrated with reference to the autocorrelation functions of (33) and (34). The only restriction is that the bandwidth $2 \delta=\beta-\alpha$ must divide the frequency range $[0, \pi)$ an integral number of times, say $q$ times. In that case, the orthogonal basis of each of the bands that partition the range will be formed by displacing the corresponding Shannon wavelet by $q$ elements at a time.

In this section, we shall look for the general conditions that are necessary to ensure that the displaced wavelet sequences are mutually orthogonal. The conditions of orthogonality will be stated in terms of the frequency-domain energy function and its square root, which is the Fourier transform of the time-domain wavelet function.

To avoid unnecessary complexity, we shall deal in terms of the continuous frequency-domain function rather the sampled version, which has been the subject of section 5. Except in cases where the energy function has an absolute discontinuity or a saltus, as in the case of the boxcar function associated with Shannon wavelets, the results can be applied without hesitation to the sampled function.

We may begin, in this section, by considering the first of the prime numbers which is $q=2$, which is the case of the dyadic wavelets. This is the only even prime number; and, therefore, it demands special treatment. In the next section, we shall deal with the case where $q$ is any other prime number, beginning with the triadic case, where $q=3$. This is a prototype for all other cases.

Ignoring subscripts, let $\xi(t) \longleftrightarrow \xi(\omega)$ denote the autocorrelation function, which may belong equally to a scaling function or to a wavelet, together with its Fourier transform, which is the corresponding energy spectrum. Then, the condition of orthogonality is that

$$
\xi(2 t)= \begin{cases}\xi_{0}, & \text { if } t=0 \\ 0, & \text { if } t \neq 0\end{cases}
$$

which is to say that $\xi(2 t)=\xi_{0} \delta(t)$, where $\delta(t)$ is the unit impulse function in the time domain. The transform of the impulse function is a constant function in the frequency domain: $\delta(t) \longleftrightarrow 1$. To see what this implies 
for the energy spectrum, define $\lambda=2 \omega$ and use the change of variable technique to give

$$
\begin{aligned}
\xi(2 t) & =\frac{1}{2 \pi} \int_{-\pi}^{\pi} \xi(\omega) e^{\mathrm{i} \omega(2 t)} d \omega \\
& =\frac{1}{4 \pi} \int_{-2 \pi}^{2 \pi} \xi(\lambda / 2) e^{\mathrm{i} \lambda t} d \lambda \\
& =\frac{1}{4 \pi} \int_{-2 \pi}^{-\pi} \xi(\lambda / 2) e^{\mathrm{i} \lambda t} d \lambda+\frac{1}{4 \pi} \int_{-\pi}^{\pi} \xi(\lambda / 2) e^{\mathrm{i} \lambda t} d \lambda+\frac{1}{4 \pi} \int_{\pi}^{2 \pi} \xi(\lambda / 2) e^{\mathrm{i} \lambda t} d \lambda .
\end{aligned}
$$

But the Fourier transform of the sequence $\xi(2 t)$ is a periodic function with one cycle in $2 \pi$ radians. Therefore, the first integral must be translated to the interval $[0, \pi]$, by adding $2 \pi$ to the argument, whereas the third integral must be translated to the interval $[-\pi, 0]$, by subtracting $2 \pi$ from the argument. After their translation, the first and the third integrands combine to form the segment of the function $\xi(\pi+\lambda / 2)$ that falls in the interval $[-\pi, \pi]$. The consequence is that

$$
\xi(2 t)=\frac{1}{4 \pi} \int_{-\pi}^{\pi}\{\xi(\lambda / 2)+\xi(\pi+\lambda / 2)\} e^{\mathrm{i} \lambda t} d \lambda .
$$

This relationship can be denoted by $\xi(2 t) \longleftrightarrow \frac{1}{2}\{\xi(\lambda / 2)+\xi(\pi+\lambda / 2)\}$. The necessary condition for the orthogonality of the displaced wavelet sequences is that the Fourier transform on the RHS is a constant function. In that case, the argument $\lambda / 2$ can be replaced by $\omega$, and the condition becomes

$$
\{\xi(\omega)+\xi(\pi+\omega)\}=c,
$$

where $c$ is a constant.

It will be observed that, if $\xi(\omega)=\xi_{1 / 2}(\omega)$ stands for energy spectrum of the dyadic scaling function, then $\xi(\omega+\pi)=\xi_{2 / 2}(\omega)$ will be the energy spectrum of the wavelet. The condition $\xi_{1 / 2}(\omega)+\xi_{2 / 2}(\omega)=1$, which actually prevails, corresponds to the conservation of energy. Pairs of filters for which the squared gains satisfy the condition are called quadrature mirror filters.

Example: The Triangular Energy Function. Consider the periodic energy functions defined over the frequency interval $[-\pi, \pi]$ by

$$
\begin{aligned}
& \xi_{1 / 2}(\omega)= \begin{cases}1-|\omega| / \pi, & \text { if }|\omega| \in[0, \pi) ; \\
0, & \text { if } \omega= \pm \pi,\end{cases} \\
& \xi_{2 / 2}(\omega)= \begin{cases}|\omega| / \pi, & \text { if }|\omega| \in[0, \pi / 2) ; \\
1 / 2, & \text { if } \omega= \pm \pi,\end{cases}
\end{aligned}
$$

Here $\xi_{1 / 2}(\omega)$ is a triangle that results from the autoconvolution in the frequency domain of the box function $\phi_{(1)}(\omega)$ of $(3)$, whilst $\xi_{2 / 2}(\omega)$ is a 
version translated by $\pi$ radians. It is manifest that these functions obey the condition of $(40)$, since $\xi_{1 / 2}(\omega)+\xi_{2 / 2}(\omega)=1$.

The Fourier transforms are given by

$$
\begin{aligned}
& \xi_{1 / 2}(t)=\left\{\frac{\sin (\pi t / 2)}{\pi t}\right\}^{2}, \\
& \xi_{2 / 2}(t)=\cos (\pi t) \xi_{1 / 2}(t) .
\end{aligned}
$$

Here, $\xi_{1 / 2}(t)$ is the square of the sinc function, whereas $\xi_{2 / 2}(t)$ is the result of a frequency shifting operation applied to $\xi_{1 / 2}(t)$.

Example: The Chamfered Box. A generalisation of the function $\xi_{1 / 2}(\omega)$ of (41), which also obeys the condition of (40), is one that can be described as a chamfered box or a split triangle, and which is defined by

$$
\xi_{1 / 2}(\omega)= \begin{cases}1, & \text { if }|\omega| \in(0, \pi / 2-\epsilon), \\ 1-\frac{|\omega+\epsilon-\pi / 2|}{2 \epsilon}, & \text { if }|\omega|=(\pi / 2-\epsilon, \pi / 2+\epsilon), \\ 0, & \text { otherwise. }\end{cases}
$$

Setting $\epsilon=\pi / 2$ reduces this to the triangular function of (41). Also subsumed under the sampled version of the present function is the sampled version of the boxcar energy function, in which the problem caused by the discontinuity at the cut-off point is handled, in effect, by chamfering the edge. (When the edge of the box is chamfered in the slightest degree, the two function values at the point of discontinuity, which are zero and unity, will coincide at a value of one half.)

A function that has the same Fourier transform as the chamfered box can be formed from the difference of two triangle functions. The first triangle is defined in the frequency domain by

$$
\Lambda_{1}(\omega)= \begin{cases}\frac{1}{2}\left(\frac{\pi}{2 \epsilon}+1\right)-\frac{|\omega|}{2 \epsilon}, & \text { if }|\omega| \in(0, \pi / 2+\epsilon), \\ 0, & \text { otherwise. }\end{cases}
$$

The Fourier transform is

$$
\Lambda_{1}(t)=\left\{\frac{\sin \{(\pi / 2+\epsilon) t\}}{\pi t}\right\}^{2},
$$

The second triangle is defined by

$$
\Lambda_{2}(\omega)= \begin{cases}\frac{1}{2}\left(\frac{\pi}{2 \epsilon}-1\right)-\frac{|\omega|}{2 \epsilon}, & \text { if }|\omega| \in(0, \pi / 2-\epsilon), \\ 0, & \text { otherwise. }\end{cases}
$$




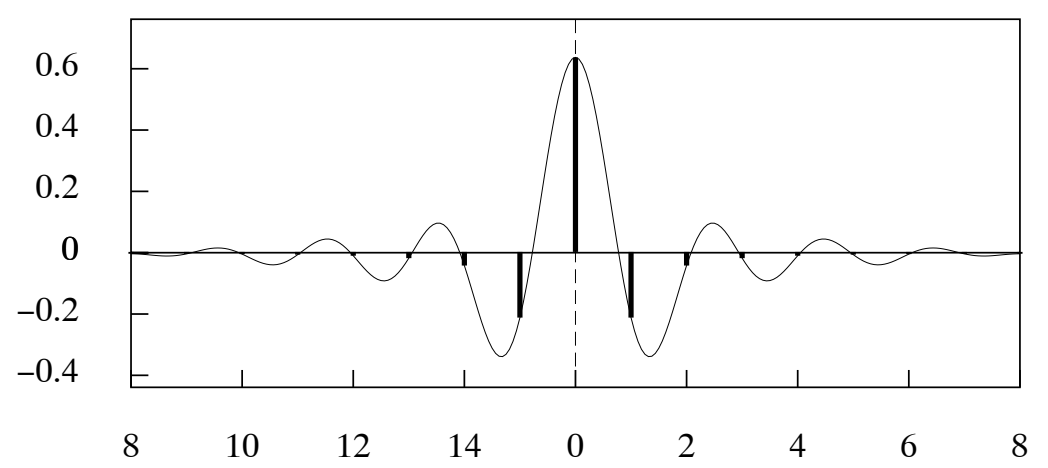

Figure 16. A circulant wavelet sequence on 16 points corresponding to a cosine bell energy function.

The Fourier transform for this one is

$$
\Lambda_{2}(t)=\left\{\frac{\sin \{(\pi / 2-\epsilon) t\}}{\pi t}\right\}^{2} .
$$

The Fourier transform of the function of $\xi_{1 / 2}(\omega)$ of $(43)$ is

$$
\xi_{1 / 2}(t)=\Lambda_{1}(t)-\Lambda_{2}(t)
$$

In the example above, the autocorrelation functions fulfil the orthogonality condition of (40) by virtue of their anti-symmetry in the vicinity of the cut-off values $\omega_{c}= \pm \pi / 2$. For a sine wave the condition of antisymmetry is expressed in the identity $\sin (-\omega)=-\sin (\omega)$. For the energy functions, the points of symmetry have the coordinates $\left(\omega_{c}, 0.5\right)$ and the conditions of anti-symmetry, which prevail in the intervals $\left(\omega_{c}-\epsilon, \omega_{c}+\epsilon\right)$, where $\epsilon \leq \pi / 2$, are expressed in the identity

$$
0.5-\xi\left(\omega_{c}-\omega\right)=\xi\left(\omega_{c}+\omega\right)-0.5 \quad \text { for } \quad \omega \in(-\epsilon, \epsilon)
$$

We may describe this as the condition of sigmoid anti-symmetry, or of $S$ symmetry for short. The terminology is suggested by the following example which uses an ordinary cosine in constructing the autocorrelation function.

Example: The Cosine Bell. The cosine bell, with a period of $2 \pi$, is defined in the frequency domain by

$$
\xi_{1 / 2}(\omega)=0.5\{1+\cos (\omega)\}
$$

It is $S$-symmetric about the point $\pi / 2$ in the frequency interval $(0, \pi)$ and about the point $-\pi / 2$ in the frequency interval $(-\pi, 0)$. The function is 
not band-limited in frequency domain - but it is band-limited in the time domain. The Fourier transform of the continuous periodic function is the three-point sequence $\{0.25,0.5,0.25\}$, which can be recognised as the autocorrelation function of the discrete Haar scaling function. The transform of the continuous periodic function $\xi_{2 / 2}(\omega)=0.5\{1-\cos (\omega)\}$ is the sequence $\{-0.25,0.5,-0.25\}$, which can be recognised as the autocorrelation function of the discrete Haar wavelet.

The Haar wavelet, which is the one with the minimum temporal dispersion, is defined, in discrete terms, on two points by

$$
\psi(t)= \begin{cases}0.5, & \text { if } t=0, \\ -0.5, & \text { if } t=1, \\ 0, & \text { otherwise. }\end{cases}
$$

The accompanying scaling function is

$$
\phi(t)= \begin{cases}0.5, & \text { if } t=0,1, \\ 0, & \text { otherwise. }\end{cases}
$$

Now consider a sequence of ordinates sampled from the energy function $\xi_{2 / 2}(\omega)=0.5\{1-\cos (\omega)\}$ at the Fourier frequencies $\omega_{j}=2 \pi / T ; j=$ $0,1, \ldots, T-1$, which extend over the interval $[0,2 \pi)$. The sequence will be real-valued and even such that $\xi_{2 / 2}\left(\omega_{j}\right)=\xi_{2 / 2}\left(\omega_{T-j}\right)$. The Fourier transform of these ordinates will, likewise, be a real-valued even sequence of $T$ points of the form $\{0.5,-0.25,0, \ldots, 0,-0.25\}$. This can be envisaged either as a single cycle of a periodic function or as a set of points distributed evenly around a circle of circumference $T$. The sequence constitutes the circular autocorrelation function of a wrapped Haar wavelet.

The Haar wavelet is not an even function. To derive a wavelet that is real and even and which has the same autocorrelation function as the wrapped Haar wavelet, we must transform into the time domain the square roots of the ordinates sampled from the cosine bell energy function. An example of such a wavelet is provided by Figure 16 .

Example: The Split Cosine Bell. A derivative of the cosine bell, which is band-limited in the frequency domain, is provided by the split cosine bell. This has a horizontal segment interpolated at the apex of the bell which, consequently, must show a more rapid transition in the vicinities of $\pm \pi / 2$.

$$
\xi_{1 / 2}(\omega)= \begin{cases}1, & \text { if }|\omega| \in(0, \pi / 2-\epsilon) ; \\ 0.5\left[1+\cos \left\{\frac{\pi}{2 \epsilon}|\omega+\epsilon-\pi / 2|\right\}\right], & \text { if }|\omega| \in(\pi / 2-\epsilon, \pi / 2+\epsilon), \\ 0, & \text { otherwise }\end{cases}
$$




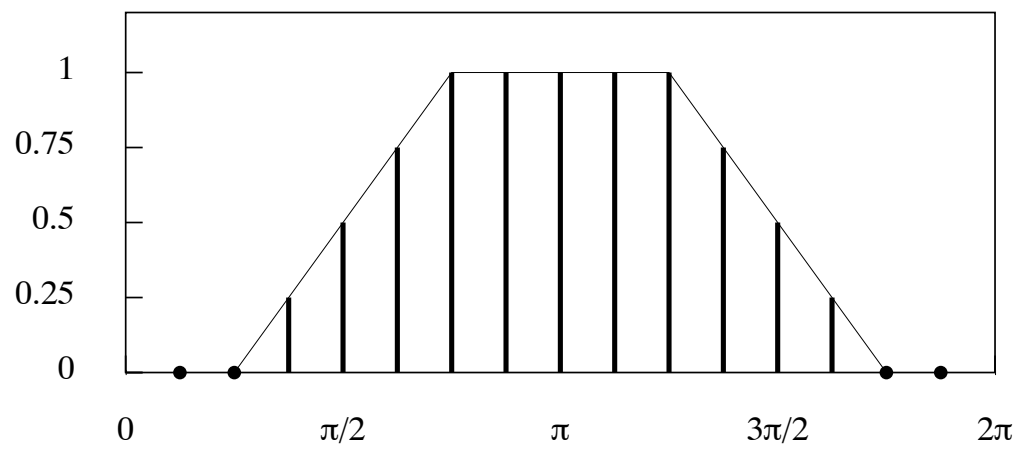

Figure 17. A sampled energy function of 16 points in the shape of a chamfered box.

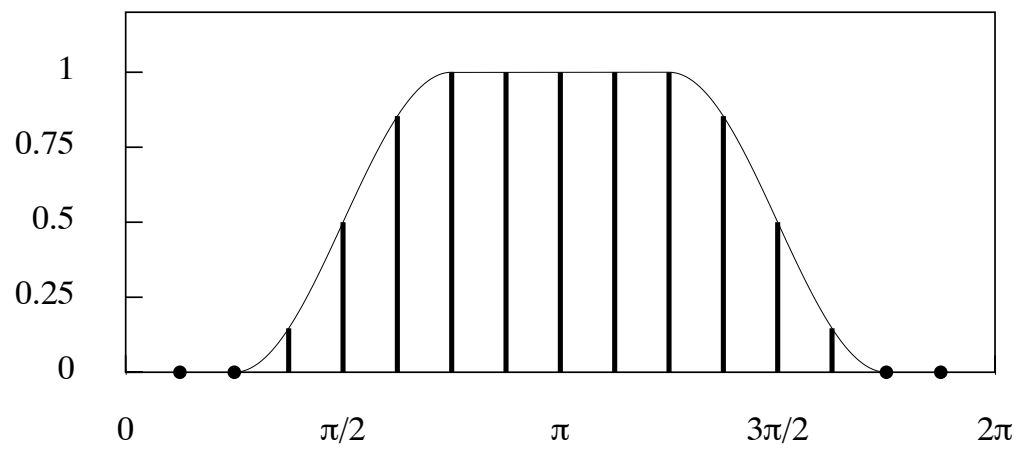

Figure 18. A sampled energy function of 16 points in the shape of a split cosine bell.

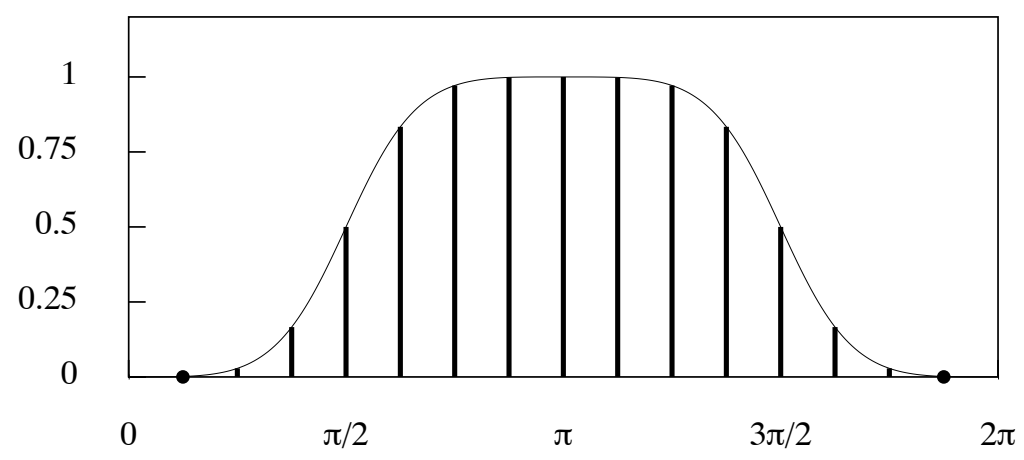

Figure 19. A sampled energy function of 16 points defined by a Butterworth function. 


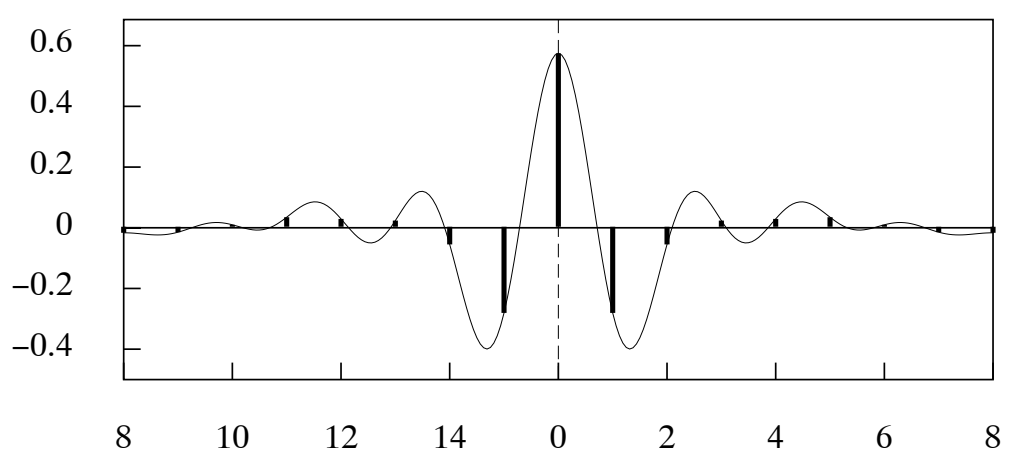

Figure 20. A circulant wavelet sequence on 16 points corresponding to the energy function of Figure 17, which is in the shape of a chamfered box.

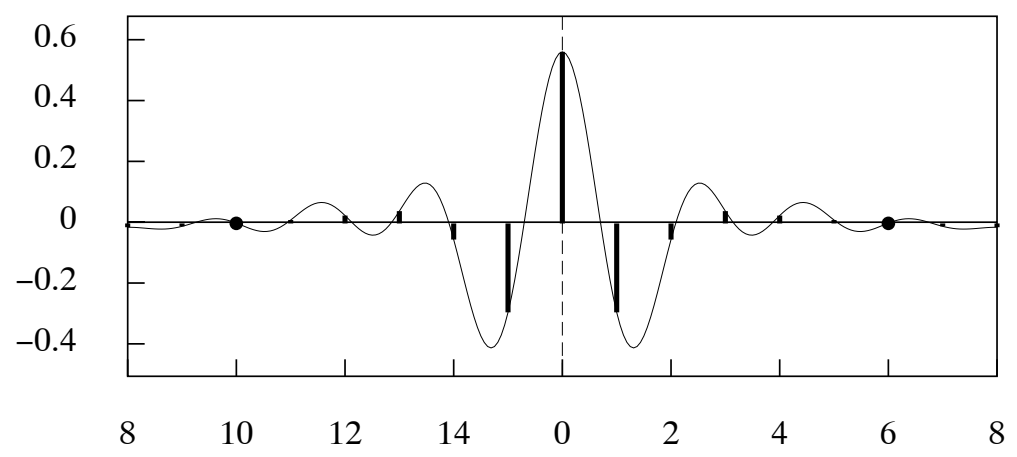

Figure 21. A circulant wavelet sequence on 16 points corresponding to the split cosine bell energy function of Figure 18.

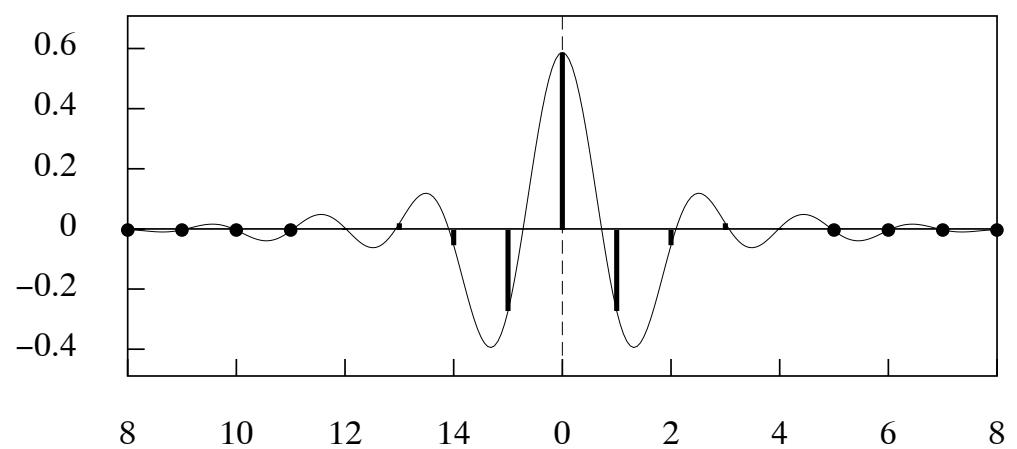

Figure 22. A circulant wavelet sequence on 16 points corresponding to the Butterworth energy function with $n=2$ of Figure 19. 
Setting $\epsilon=\pi / 2$ reduces this to the cosine bell of (50).

Observe that, if $\epsilon$ divides $\pi$ an even number of times, then the split cosine bell can be expressed as a sum of cosine bells, each of width $4 \epsilon$, at displacements relative to each other that are multiples of $2 \epsilon$. In that case, the Fourier transform of the function has a particularly simple analytic expression.

The split cosine bell has been advocated by Bloomfield (1976) as a means of truncating and tapering the time-domain coefficients of an ideal bandpass filter to create a practical FIR filter. Its advantage over the chamfered box in this connection lies in the fact that it possesses a first derivative that is continuous everywhere. Its avoidance of discontinuities reduces the spectral leakage. It is therefore to be expected that a wavelet derived from a cosine bell energy function will have a lesser temporal dispersion than one that has been derived from the corresponding chamfered box.

Example: The Butterworth Function. Another family of energy functions from which the wavelets may be derived is provided by the function that defines the frequency response of a digital Butterworth filter with a cut-off point at $\omega=\pi / 2$ :

$$
\begin{aligned}
& \xi_{1 / 2}(\omega)=\left(1+\{\tan (\omega / 2)\}^{2 n}\right)^{-1}, \\
& \xi_{2 / 2}(\omega)=1-\xi_{1 / 2}(\omega) .
\end{aligned}
$$

When $n=1$, the Butterworth function is the square of a cosine. Increasing the value of $n$ increases the rate of the transition between the pass band and the stop band of the filter, such that the function converges to the boxcar function $\phi_{(1)}(\omega)$ of (3) - see Pollock (1999), for example.

In the context of the Butterworth digital filter, the integer parameter $n$ represents the degree of a polynomial operator. In the present context, there is no reason why $n$ should be restricted to take integer values. It will be found, for example, that, when $n=0.65$, the Butterworth function provides a close approximation to the triangular energy function of (41). This is shown in Figure 23 together with the effects of other values of the parameter.

The Butterworth function, which satisfies the condition of $S$-symmetry, appears to be preferable to the split cosine bell. The relative merits of various families of wavelets proposed in this section can be assessed with reference to Figures 17-22, which show the energy functions together with the wavelets that are derived from them.

A remarkable feature of the Butterworth wavelet is that, beyond a short distance from the central point, where $t=0$, the ordinates are virtually zeros. The virtual zeros are indicated in Figure 22 by black dots, the first of which corresponds to a value of $\psi(t=6)=-0.00096$. Moreover, such 


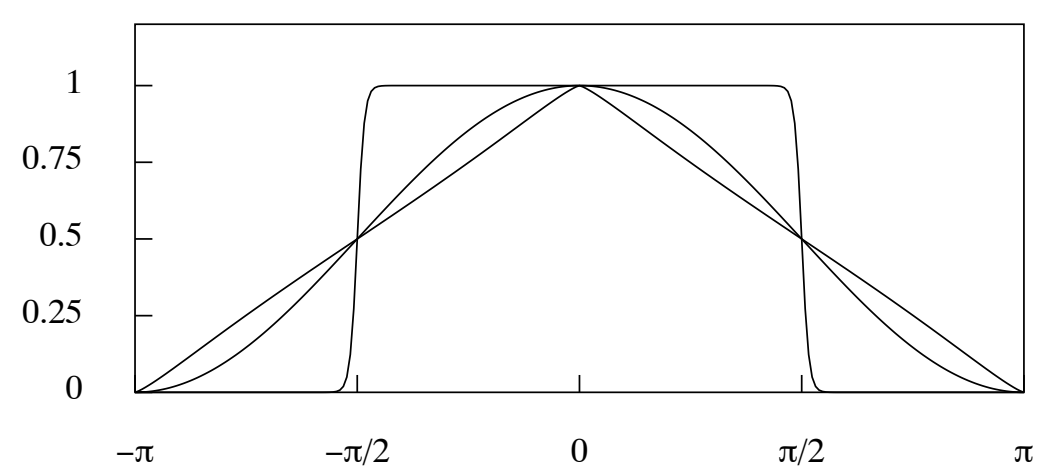

Figure 23. The Butterworth function with the parameter values $n=0.62$ (the triangle), $n=1$ (the bell) and $n=20$ (the boxcar).

values are reduced as $T$ increases and as the wavelet is wrapped around a widening circle.

One might recall the fact that, for a non-circulant wavelet on a finite support, the condition of sequential orthogonality necessitates an even number of points - see, for example, Percival and Walden (2000, p.69). This precludes the symmetry of the coefficients about a central value. Nevertheless, the Butterworth wavelet, which satisfies the orthogonality conditions, has virtually a finite support and is also symmetric.

\section{Conditions of Orthogonality in the Non-dyadic Case}

We shall now consider the general case where the wavelets subsist in $q$ bands within the frequency interval $[0, \pi]$, where $q$ is a prime number. We shall begin by considering the triadic case where $q=3$. This serves as a prototype for all other cases. First, it is necessary to indicate the manner in which the triadic wavelets may be constructed from various $S$-symmetric energy functions, such as those that have been considered in the previous section.

Consider an energy function $B(\omega)$ defined on the interval $[-\pi, \pi]$ that corresponds to a dyadic scaling function or, equally, to a half-band lowpass filter with a nominal cut-off frequency of $\pi / 2$. This function can be mapped, via a compression of the frequency axis, onto an interval of length $2 \pi / 3$. The effect is achieved by multiplying the frequency argument by a factor of 3 to give $B(3 \omega) ; \omega \in[-\pi / 3, \pi / 3]$.

To construct the triadic lowpass wavelets, for which the nominal range of the energy function is the interval $(-\pi / 3, \pi / 3)$, copies of $B(3 \omega)$ are placed at the centres $-\pi / 6$ and $\pi / 6$. The result is the function defined on the 

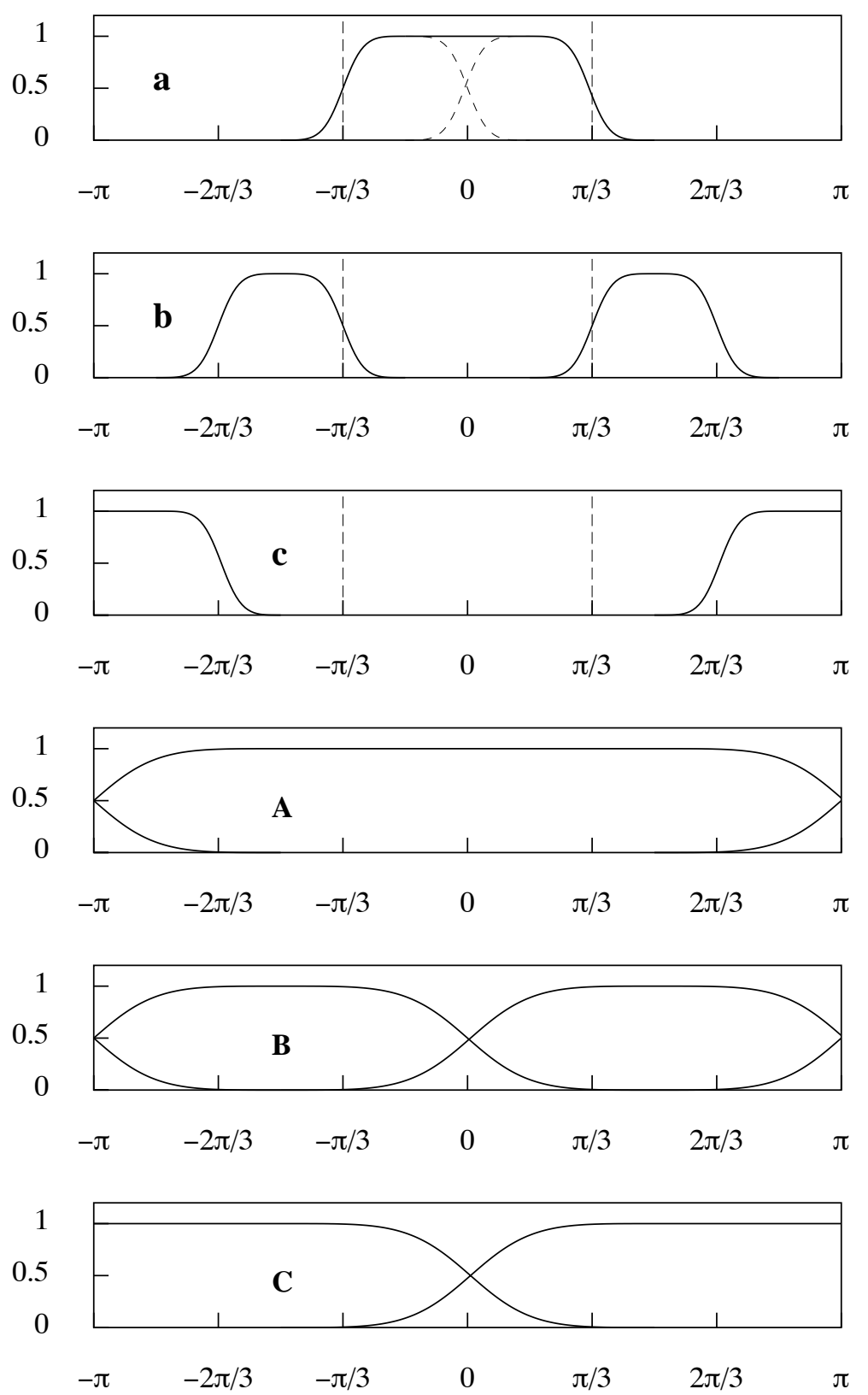

Figure 24. The triadic energy functions, figures a-c. The segments of the latter, which are demarcated by the dotted lines and which are each of length $2 \pi / 3$, are dilated by a factor of 3 and overlaid on the interval $[-\pi, \pi]$ to form figures $\mathrm{A}-\mathrm{C}$. 
interval $[-\pi, \pi]$ by

$$
\xi_{1 / 3}(\omega)= \begin{cases}B(3 \omega+\pi / 6)+B(3 \omega-\pi / 6), & \text { if } \omega \in[-\pi / 2, \pi / 2], \\ 0, & \text { otherwise. }\end{cases}
$$

Figure 24a shows the manner in which the copied functions are fused together.

To construct the triadic bandpass wavelet, for which the nominal pass band is the interval $(\pi / 3,2 \pi / 3)$, the two copies of $B(3 \omega)$ are translated to centres at $\pi / 2$ and $-\pi / 2$ and combined to give

$$
\xi_{2 / 3}(\omega)= \begin{cases}B(3 \omega+\pi / 2), & \text { if } \omega \in[-5 \pi / 6,-\pi / 6], \\ B(3 \omega-\pi / 2), & \text { if } \omega \in[5 \pi / 6, \pi / 6], \\ 0, & \text { otherwise. }\end{cases}
$$

The result is shown in Figure 24b.

In the case of the triadic highpass wavelet, for which the nominal pass band is the interval $(2 \pi / 3, \pi)$, the two copies of $B(3 \omega)$ are translated to centres as $5 \pi / 6$ and $-5 \pi / 6$ to give

$$
\xi_{3 / 3}(\omega)= \begin{cases}B(3 \omega+5 \pi / 6)+B(3 \omega-5 \pi / 6), & \text { if } \omega \in[-\pi / 2, \pi / 2] \\ 0, & \text { otherwise. }\end{cases}
$$

This can also be obtained simply by translating the centre of $\xi_{1 / 3}(\omega)$ from $\omega=0$ to $\omega= \pm \pi$. The feature becomes fully apparent only when the interval $[-\pi, \pi]$ is wrapped around the circle such that $\pi$ and $-\pi$ coincide at the point diametrically opposite the point where $\omega=0$. The result is shown in Figure 24c in terms of the linear interval.

Let $\xi(t) \longleftrightarrow \xi(\omega)$ denote the autocorrelation function of any one of the triadic wavelets together with the energy function, which is its Fourier transform. Then, the relevant condition of sequential orthogonality is that $\xi(3 t)=0$ if $t \neq 0$. Define $\lambda=3 \omega$. Then,

$$
\begin{aligned}
\xi(3 t) & =\frac{1}{2 \pi} \int_{-\pi}^{\pi} \xi(\omega) e^{\mathrm{i} \omega(3 t)} d \omega \\
& =\frac{1}{6 \pi} \int_{-3 \pi}^{3 \pi} \xi(\lambda / 3) e^{\mathrm{i} \lambda t} d \lambda \\
& =\frac{1}{6 \pi}\left\{\int_{-3 \pi}^{-\pi} \xi(\lambda / 3) e^{\mathrm{i} \lambda t} d \lambda+\int_{-\pi}^{\pi} \xi(\lambda / 3) e^{\mathrm{i} \lambda t} d \lambda+\int_{\pi}^{3 \pi} \xi(\lambda / 3) e^{\mathrm{i} \lambda t} d \lambda\right\} \\
& =\frac{1}{6 \pi} \int_{-\pi}^{-\pi} \sum_{j=-1}^{1} \xi([2 \pi j+\lambda] / 3) e^{\mathrm{i} \lambda t} d \lambda .
\end{aligned}
$$


It follows that

$$
\xi(3 t) \longleftrightarrow \sum_{j=-1}^{1} \frac{1}{3} \xi([2 \pi j+\lambda] / 3)=\delta(\omega) .
$$

The condition of sequential orthogonality is that $\delta(\omega)$ must be a constant function such that its Fourier transform is the unit impulse. Figures $24(\mathrm{~A}-$ C) show how the segments of the three energy functions that are demarcated by the dotted lines are dilated and overlaid on the interval $[-\pi, \pi]$. In each case, adding the segments produces the constant function $\delta(\omega)=c$. The overlaying of the segments occurs when each is wrapped around the same circle of circumference $2 \pi$. In fact, the segments need not be separated one from another. They can be wrapped around the circle in one continuous strip.

The condition of lateral orthogonality cannot be satisfied by wavelets in adjacent frequency bands. This a consequence of the spectral leakage from each band into the neighbouring bands. However, in the present triadic specification, which interpolates a third band between the lowpass and highpass bands and which limits the extent of the leakage on either side to one half of the nominal bandwidth, conditions of lateral orthogonality prevail between non-adjacent bands.

Now let us consider a bandpass filter with a nominal width of $\pi / 3$ centered, in the positive frequency range, on some point $\theta \in[\pi / 6,5 \pi / 6]$ that lies between the centres of the lowpass and the highpass filters. The energy function of the filter is specified over the interval $[-\pi, \pi]$ by

$$
\xi_{\theta / 3}(\omega)= \begin{cases}B(3 \omega+\theta) & \text { if } \omega \in[\theta-\pi / 3, \theta+\pi / 3] \\ B(3 \omega-\theta), & \text { if } \omega \in[-\pi / 3-\theta, \pi / 3-\theta] \\ 0, & \text { otherwise. }\end{cases}
$$

It can be shown that, regardless of the actual value taken by $\theta$ within the designated range, the condition $\xi_{\theta / 3}(6 t)=0$ prevails for all $t \neq 0$, which is to say that wavelets within the band that are separated by multiples of 6 points are mutually orthogonal.

To demonstrate this, we must consider the decomposition $\xi_{\theta / 3}(\omega)=$ $\xi_{\theta / 3}^{+}(\omega)+\xi_{\theta / 3}^{-}(\omega)$, where $\xi_{\theta / 3}^{+}(\omega)$ has a zero segment in place of the segment of $B(3 \omega-\theta)$, and where $\xi_{\theta / 3}^{-}(\omega)$ has a zero segment in place of the segment of $B(3 \omega+\theta)$. Since $\theta$ is arbitrary, the interaction of $\xi_{\theta / 3}^{+}(\omega)$ and $\xi_{\theta / 3}^{-}(\omega)$ is undetermined, and we must treat the two functions separately.

In order that $\xi_{\theta / 3}^{+}(\omega)$ should generate a uniform function over the interval $[-\pi, \pi]$ and beyond, it must be dilated by a factor of 6 before being wrapped around the circle. Then, the pass band, which has a (nominal) 


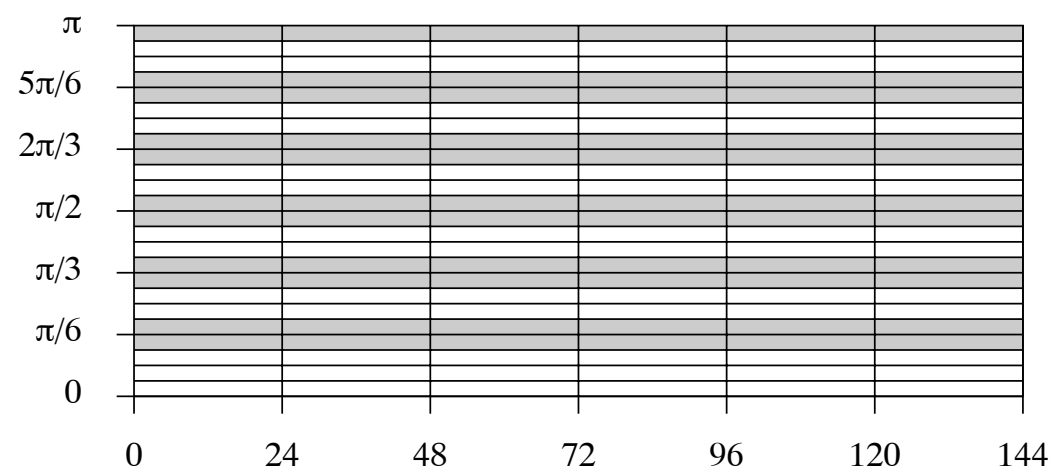

Figure 25. The time-frequency plane for 144 data points partitioned with 24 frequency intervals and 6 time periods.

width of $\pi / 3$, will acquire a width of $2 \pi$, which is sufficient to encompass the circle with a band of constant height. Equivalent conditions apply to $\xi_{\theta / 3}^{-}(\omega)$. The upshot is that the wavelets that lie within a pass band of width $\pi / 3$, located at an arbitrary centre, are mutually orthogonal when separated by multiples of 6 points.

The generalisations of the analysis of this section from $q=3$ to cases of other integers is immediate. For the case where the interval $[0, \pi]$ is divided in $q>2$ bands of equal width, the condition for the sequential orthogonality of wavelets separated by $q$ points is that

$$
\xi(q t) \longleftrightarrow \sum_{j=(1-q) / 2}^{(q-1) / 2} \frac{1}{q} \xi([2 \pi j+\lambda] / q)=c .
$$

For a band of width $\pi / q$, with $q \geq 2$, centred on an arbitrary point $\theta$ within $[\pi / 2 q, \pi-\pi / 2 q]$, the proof that wavelets separated by multiples $2 q$ points are mutually orthogonal in indicated by the proof for the case where $q=3$. We shall conclude the paper with an example that shows of how these conditions can be used in the analysis of the finite data sequence that was described in the introduction.

Example. Figure 25 shows the time-frequency plane for 144 data points, partitioned in a manner that is appropriate to the analysis of the monthly airline passenger data of Figure 5. The bands that have been highlighted cover the spectral structure of the seasonal fluctuations that is revealed by the periodogram of Figure 6. On either side of the the seasonal frequencies $\{\pi j / 6 ; j=1, \ldots, 5\}$, there are adjacent bands of $7 \frac{1}{2}$ degrees in width. Altogether, there are 24 bands of equal width dividing the frequency range, 
and the time span of the sample is divided into six sections, each of which spans a two-year period.

With this partitioning, it should be possible to reveal the evolution of the seasonal pattern by showing the progression of the amplitude coefficients of the wavelets within the highlighted bands. In testing the statistical null hypothesis of temporal homogeneity, which is liable to be rejected, it is helpful to have wavelets that are mutually orthogonal.

The interstices between the highlighted bands are effective in ensuring the lateral orthogonality of the wavelets, whenever they are derived from one of the templates that have been provided in Section 6. However, the wavelets in the contiguous bands that fall on either side of the frequencies $\{\pi j / 6 ; j=1, \ldots, 5\}$ will not be mutually orthogonal. This problem can be overcome by combining these bands. The combined bands will be populated by twice as many wavelets as the original narrower bands. However, the distances that separate orthogonal wavelets will remain the same at 24 points.

\section{References}

Bloomfield, P., (1976), Fourier Analysis of Time Series: An Introduction, John Wiley and Sons, New York.

Boggess, A., and F.J. Narcowich, (2001), A First Course in Wavelets with Fourier Analysis, Prentice-Hall, Upper Saddle River, New Jersey.

Box, G.E.P., and G.M. Jenkins, (1976), Time Series Analysis: Forecasting and Control, Revised Edition, Holden-Day, San Francisco.

Burrus, C.S, R.A. Gopinath and H. Guo, (1998), Introduction to Wavelets and Wavelet Transforms: A Primer, Prentice-Hall, Upper Saddle River, New Jersey.

Daubechies, I., (1988), Orthonormal Bases of Compactly Supported Wavelets, Communications in Pure and Applied Mathematics, 41, 909-996.

Daubechies, I., (1992), Ten Lectures on Wavelets, Society for Industrial and Applied Mathematics, Philadelphia.

Dirac, P.A.M., (1958), The Principles of Quantum Mechanics, Fourth Edition, Oxford University Press, Oxford.

Gopinath, R.A., and C.S. Burrus, (1993), Wavelet Transforms and Filter Banks. In C.K. Chui, editor, Wavelets: A Tutorial in Theory and Applications, 603-655, Academic Press, San Diego. Volume 2 of Wavelet Analysis and its Applications.

Mallat, S.G., (1989), A Theory for Multiresolution Signal Decomposition: The Wavelet Representation, IEEE Transactions on Pattern Analysis and Machine Intelligence, 11, 674-693.

Misiti, M., Y. Misiti, G. Oppenheim and J-M. Poggi, (1997), Wavelet Toolbox for Use with MATLAB, The MathWorks Inc., Natick, Massachusetts.

Nason, G.P., and R. von Sachs, (1999), Wavelets in Time Series Analysis, Philosophical Transactions of the Royal Society of London, Series A, 357, 2511-2526.

Nason, G.P., and T. Sapatinas, (2002), Wavelet Packet Transfer Function Modelling of Nonstationary Time Series, Statistics and Computing, 12, 45-56.

Newland, D.E., (1993), An Introduction to Random Vibrations, Spectral Analysis and Wavelets: 3rd edition, Longman Scientific and Technical, Harlow, Essex. 
Percival, D.B., and A.T. Walden (2000), Wavelet Methods for Time Series Analysis, Cambridge University Press, Cambridge.

Pollock, D.S.G., (1999), Time-Series Analysis, Signal Processing and Dynamics, Academic Press, London.

Pollock, D.S.G., (2002), Circulant Matrices and Time-Series Analysis, The International Journal of Mathematical Education in Science and Technology, 33, 213-230.

Ramsey, J.B., and and C. Lampart, (1998), The Decomposition of Economic Relationships by Time Scale Using Wavelets: Expenditure and Income, Studies in Nonlinear Dynamics and Econometrics, 3, 23-42.

Ramsey, J.B., (1999), The Contribution of Wavelets to the Analysis of Economics and Financial Data, Philosophical Transactions of the Royal Society of London, Series A, 357, 2593-2606.

Shannon, C.E., and W. Weaver, (1964), The Mathematical Theory of Communication, The University of Illinois Press, Urbana, Illinois.

Steffen, P., P.N. Heller, R.A. Gopinath, and C.S. Burrus, (1993), Theory of Regular $M$-band Wavelets, IEEE Transactions on Signal Processing, 41, 3497-3511.

Strang, G., and T. Nguyen, (1997), Wavelets and Filter Banks, Prentice-Hall, Upper Saddle River, New Jersey.

Vaidyanathan, P.P., (1990), Multirate Digital Filters, Filter Banks, Polyphase Networks and Applications: A Tutorial, Proceedings of the IEEE, 78, 56-93.

Vaidyanathan, P.P., (1993), Multirate Systems and Filter Banks, Proceedings of the IEEE, 78, 56-93.

Vetterli, M., and J. Kovacević (1995), Wavelets and Subband Coding, Prentice-Hall, Upper Saddle River, New Jersey.

Wickerhauser, V.M., (1994), Adapted Wavelet Analysis from Theory to Software, A.K. Peters, Natick, Massachusetts.

Woodward, P.M., (1953), Probability and Information Theory with Applications to Radar, McGraw-Hill Book Company, New York. 
